\title{
Chemicals from Coal Coking
}

Marcos Granda ${ }^{\dagger}$, Clara Blanco ${ }^{\dagger}$, Patricia Alvarez ${ }^{\dagger}$, John W. Patrick ${ }^{\ddagger}$ and R. Menéndez ${ }^{*}, \dagger$

${ }^{\dagger}$ Instituto Nacional del Carbón, CSIC. C/Francisco Pintado Fe 26. 33011-Oviedo, Spain

Faculty of Engineering, University of Nottingham. University Park. Nottingham NG7 2RD, United Kingdom

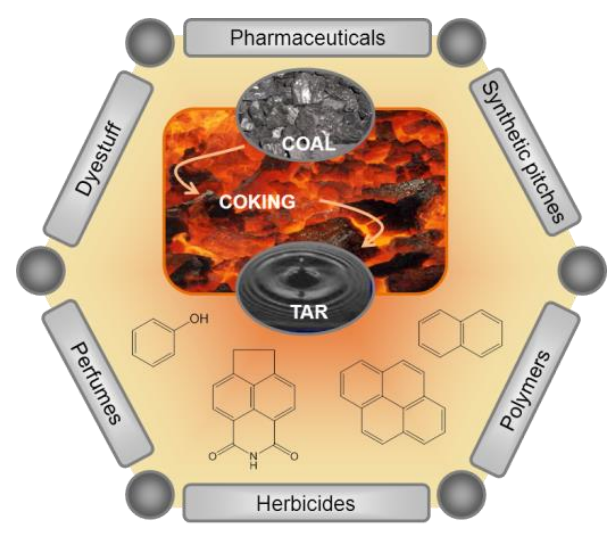

CONTENT

1. Introduction

2. The Coal Coking Process

3. Coal Coking by-Products

4. Processing of Coal Coking by-Products

4.1. Tar Distillation

4.2. Coke Oven Gas Treatment 
4.3. Other Products

5. Chemicals from Tar Distillation Fractions

\subsection{BTX Fraction}

5.2. Carbolic Oil

5.3. Naphthalene Oil

5.4. Wash Oil

5.5. Anthracene Oil

5.6. Coal-Tar Pitch

6. Polymers from Coal Coking Chemicals

6.1. Xylene and Naphthalene Derivatives as Intermediates in the Production of Polymers

6.2. Xylene-Based Polymers

6.2.1. Polyesters

6.2.2. Aramids

6.2.3. Benzobisoxazoles

6.3. Naphthalene-Based Polymers

6.4. Phenol-Based Polymers

6.4.1. Polycarbonates 


\subsubsection{Phenolic Resins}

7. Synthetic Pitches from Coal Coking Chemicals

7.1. The Carbonization Process. Mechanisms

7.2. Pitches from Single Polycyclic Aromatic Hydrocarbons

7.3. Pitches from Mixtures of Polycyclic Aromatic Hydrocarbons

8. FUTURE PROSPECTS

AUTHORS' INFORMATION

Corresponding Author

Notes

Biographies

ACKNOWLEDGMENTS

REFERENCES 


\section{INTRODUCTION}

The term coal covers a wide range of materials from brown coals or lignites to bituminous coals and anthracites, but all of them can be roughly described as having complex macromolecular organic structures. These structures are composed predominantly of carbon with significant proportions of oxygen and hydrogen and small percentages of nitrogen and sulphur. This elemental composition makes coal a storehouse of chemicals and even more so if the presence of valuable minor and trace elements associated with the coal are also taken into account.

The problem is to find the key to this storehouse and to unlock it so as to be able to utilise the elements present in order to produce more valuable substances which will provide the feedstocks or reaction intermediaries for the production of substances and materials which contribute to the desired life style of today.

The route from coal to chemicals can be simply apportioned into the three processes of coal carbonization, coal gasification ${ }^{1}$ and coal liquefaction ${ }^{2}$, this review being concerned with the first of them. The origins of the chemical industry can be traced back to coal carbonization for the purpose of producing coal tar, but the developments of most significance for the chemical industry were the production of coal gas for the purpose of illumination and the coking of coal (i.e., the conversion process by means of which coal is transformed into a graphitizable material called coke) in by-product recovery ovens to produce metallurgical quality coke. Both of these processes yielded coal tar from which a host of chemicals necessary for an expanding organic chemical industry could be separated. 
The history of the chemicals derived from coal is intimately linked to the conversion of coal into coke. This transformation yields, in addition to coke, a series of by-products from which a wide variety of aromatic chemicals can be obtained ${ }^{3,4,5,6,7}$.

A patent for making pitch and tar from coal was taken out in 1681 and coal distillation took off in the following years. By 1781 the Earl of Dundonald had patented the distillation of coal with the recovery of tar, pitch, salts, coke and other products ${ }^{8}$. By this time, the coal-based chemical industry was well under way and continued to flourish right up until the mid-twentieth century9. During this period, coal became the sole source of aromatic chemicals. After the Second World War, this panorama changed drastically because of the irruption of petroleum onto the scene. Since then, petroleum has been extracted on a massive scale and the petrochemical industry has continued to develop at a vertiginous pace. This industry has come to the point where it is now able to produce chemicals, both aliphatic and aromatic, directly and in so doing has relegated the "old" carbochemical industry to the background. However, the current prospects for petroleum suggest that it would be prudent to think again about coal, for which a longer life-span has been predicted, as a source of aromatic chemicals.

Because of their highly aromatic composition, the chemicals derived from coking processes have a molecular structure that is not easy to find in chemicals obtained from other sources. At the present time, chemicals such as benzene and its derivatives (e.g., xylenes, phenols, etc.) and even naphthalene, are mainly manufactured by the petrochemical industry. These chemicals can also be obtained from coal coking fractions ${ }^{3}$, as an alternative source to petroleum. However, where the coal coking products show their supremacy is in providing polyaromatic compounds, such as anthracene, pyrene, etc. These compounds of three or four condensed aromatic rings can only be obtained from coal sources ${ }^{10}$. 
It is expected, therefore, that chemicals from the coal coking processes will become increasingly more significant and, if not in the short short-term, at least in the mid- and long-term, make coal a competitive and/or alternative source to petroleum.

The essential primary step, at the present time, in the production of organic chemicals, which is the mainstay of the chemical industry, is the distillation of crude oil to obtain a series of fractions from refinery gas, gasoline (or petroleum), naphtha, kerosene, diesel oils to bitumen, the highest boiling fraction. Naphtha provides the feedstock for the production of substances such as methanol, ethylene and propylene whilst with catalytic cracking it can also provide gasoline together with benzene, toluene, butylenes, butadienes, etc. This range of chemicals is the starting point for the production of a vast range of products including plastics such as polyethylene, polyvinyl chloride (PVC), polystyrene, synthetic rubber, antifreeze, polyester fibres, nylon and other polyamides, ethanol and detergents, as well as acetic acid, a simple but important chemical for the production of chemical intermediates, of which cellulose acetate is a good example ${ }^{11}$.

This simplified description of the chemicals obtained from crude oil is a general production route which can be varied to some extent to meet different economic conditions and consumer demands but it serves to demonstrate the range of chemicals required by the chemical industry, all of which can be derived from the by-products of the coking of coal.

It is not our intention in this review to describe in detail the technical approaches for producing chemicals from coal coking derivatives, which anyway has been extensively described by others $^{12,13,14}$. This review aims to address the production of chemicals from the point of view of their synthesis and subsequent transformation into upgraded products. To this end it describes the refining process for obtaining liquid fractions enriched in aromatic compounds, the methods used 
to separate chemicals from these fractions and their subsequent utilization in the production of polymers and synthetic pitches. The review concludes with some reflexions on the future of coal coking derivatives.

\section{THE COAL COKING PROCESS}

Bituminous coals with a carbon content of around 75-90 wt.\% (all the percentages referring to coal and coke in this review are expressed on a dry and mineral matter-free basis, dmmf) are primarily used in the coking process for the production of metallurgical coke ${ }^{15}$. When coal is heated up to $1000-1200{ }^{\circ} \mathrm{C}$ at a slow heating rate $\left(2-5{ }^{\circ} \mathrm{C} \min ^{-1}\right)$ in the absence of air, the moisture is removed before the coal organic macromolecular network start to decompose with the release of the volatile constituents (i.e., substances released from coal under any conditions of heating) and it is these volatiles that provide the chemical precursors required for the production of the final chemical products. The volatile matter content, a parameter that reflects the coalification degree of coal (coal rank), is one of the most important characteristics of bituminous coals, especially with regard to the production of metallurgical coke. But a coking coal is not entirely defined by rank. Its ability to successively soften, swell and resolidify, that is to say its plasticity during thermal treatment, is a prerequisite for producing coke (a graphitizable carbon material) and for controlling its structure and properties. Indeed, the coking process is usually divided into three temperature stages which are related to coal plasticity ${ }^{16,17,18}$ : (i) the pre-plastic stage up to $350{ }^{\circ} \mathrm{C}$, where the volatiles (i.e., carbon dioxide and light hydrocarbons) are produced mainly from evaporation rather than from the degradation of the macromolecular structure of the coal; (ii) the plastic stage from 350 and $500^{\circ} \mathrm{C}$ (which depends on rank), where 
the extensive primary degradation of the coal structure takes place resulting in the formation of condensable and uncondensable species and an intermediate solid carbon material, referred to as semi-coke; (iii) the post-plastic stage up to the final coking temperature which involves the progressive structural reorganization of a semi-coke to a high-temperature coke by evolving mainly hydrogen followed by the consolidation of the graphitizable structure ${ }^{15}$.

Nowadays to overcome the shortage of good coking coals, the coking industry works with blends of coals to meet the chemical and physical characteristics required for a coke to be used in a blast furnace. Thus, the formulation of coal blends is based on the use of several coking coals with different volatile matter contents, chemical impurities (i.e., sulphur, ashes, etc.), maceral compositions and rheological properties ${ }^{12}$.

Coke finds its main application in the iron and steel industry, as it exhibits a structure (e.g., anisotropic, porous, etc.) and a range of properties (e.g., high mechanical strength, relatively low chemical reactivity and good thermal conductivity) which satisfy the main requirements for use in iron-making: (i) as a reductant of iron oxides to pig iron in the blast furnace; (ii) as a permeable material to permit the easy drainage of iron to the bottom of the hearth and the rise of the gases to the top and (iii) as a source of heat ${ }^{19}$.

The aim of the current industrial coking strategy is to obtain the highest possible coke yield and quality to ensure optimal coke performance in the blast furnace. The quality of the coke is mainly determined by the characteristics of the coal blend, although coke-oven design and coking conditions (flue temperature, coking time, bulk density, etc.), the use of carbon-based additives and the preparation and pre-treatment of the coal blend also play relevant roles. Coke yields are usually in the range of $\sim 75-80$ wt.\%. This review will focus on the by-products 
generated in the coke production process, which depend on the inherent composition and structure of the parent coal, the coking conditions and the coking plant design. From a chemical point of view, the coal plastic stage in the coke oven is a complex process which includes multiple chemical reactions that occur simultaneously (e.g., de-alkylation, thermal cracking, dehydrogenation, condensation, hydrogen transfer, isomerization, etc. ${ }^{20,21}$ ). These chemical reactions are accompanied by physical changes as mentioned above and both processes are responsible for the formation of the chemical compounds found in tar.

\section{COAL COKING BY-PRODUCTS}

The by-products of the coal coking process, including the coke oven gas and tar, represent about $20-25$ wt.\% of the parent coal. The coke oven gas (mainly permanent gas), which accounts for $15-20$ wt.\% of the coking process, is stored in gas containers and used as fuel to heat the coke ovens and other facilities in an integrated steel installation. It is mainly composed of $\mathrm{H}_{2}$ (derived from aromatic condensation) and $\mathrm{CH}_{4}$ (from de-alkylation reactions), and, to a lesser extent, $\mathrm{CO}$, $\mathrm{CO}_{2}$ and light hydrocarbons ${ }^{22}$. Tar, another by-product of the coking activity ( $\left.\sim 3-5 \mathrm{wt} . \%\right)$, has a low specific value in the market. It has therefore attracted less attention. However, since tar is composed of hundreds of polycyclic aromatic hydrocarbons ${ }^{23,24}$, it is a feedstock of great importance for the carbochemical industry. In fact, tar is the main source of pitch and offers a wide range of chemicals and carbon materials with relevant industrial applications. By 1950 some 200 compounds had been detected in coal $\operatorname{tar}^{25}$ and since then many more of the estimated several thousand compounds have been identified by means of the modern analytical techniques 
developed in recent years, only a small number of these compounds have actually been separated (Morgan and Kandiyoti in another article in this series).

Organic vapours originated in the zone of the coke oven when coal is in the plastic stage ${ }^{26}$ (Figure 1). In this zone, vapours mix with other gases and pass through the plastic coal towards the hot zone (either semi-coke/coke or oven walls), on their way to the unoccupied space at the top of the coke oven, giving rise to primary tar (i.e., long chain of aliphatic compounds with alkyl and hydroxyl substituents, alkyl-substituted and unsubstituted aromatics and heteroatombased compounds). Once it occupies the free space, the primary tar mixes with the overheated gases formed in the coking zone and reaches temperatures above $600-700{ }^{\circ} \mathrm{C}$. Under these conditions, the components of the primary tar undergo a series of physical and chemical transformations that lead to the formation of more thermally stable species (secondary pyrolysis $)^{27}$. Thus, side chains and substituents are removed by conversion into naphthenic species and, finally, into aromatics from one to several condensed rings. Compounds containing

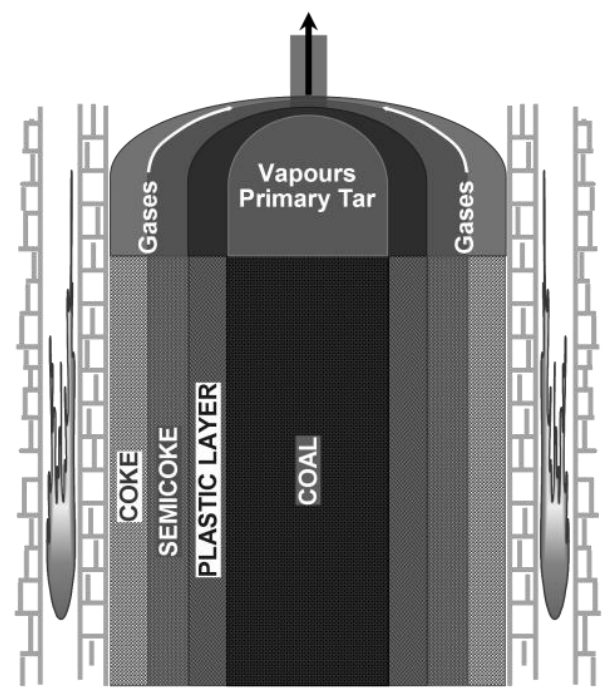

Figure 1. Longitudinal cross-section of a coking oven. 
heteroatoms, which are relatively abundant in primary tar, decompose, giving rise to $\mathrm{H}_{2} \mathrm{O}, \mathrm{H}_{2} \mathrm{~S}$, $\mathrm{HCN}$ and $\mathrm{NH}_{3}$, among others. As a result, the tar becomes more aromatic and condensed. Flue temperature is a critical factor in the final composition of the recovered condensable fraction which constitutes the high-temperature tar. The secondary pyrolysis is affected not only by the flue temperature but also by other operational parameters such as coking time, heating rate, bulk density, internal pressure as well as the design of the coke oven and the characteristics of the coal.

Currently, by-product plants in the coking industry have completely different operating sequences and recovery strategies. The main objective of this crude gas processing stage is to obtain a clean fuel gas. The crude gas generated in the ovens, including the tar, is collected from the mains and air cooled before being further cooled by a high velocity spray of coal tar and liquor. Finally, the tar is stored in tanks until it is further refined in chemical plants. In one type of by-product plant, after the tar has been removed, the gas goes to a saturator where ammonia is recovered as ammonium sulphate. Water spray cooling then causes the condensation of the water and naphthalene. Next a wash-oil scrubber recovers the crude benzole. The gas is further purified by the removal of hydrogen sulphide and hydrogen cyanide before it is collected in a gas holder $^{28}$. There are several variations of this sequential by-product recovery system. In one of them, the gases are cooled by a jet of ammoniacal liquor immediately after being collected from the ascension pipes. This causes the condensation of the tar and aqueous solution of ammonium salts after which the tar is decanted prior to further refinement.

The process just described leads to the separation of gas, tar, crude benzole, ammoniacal liquor, and naphthalene. The products evolved and the proportions of the various volatile species are dependent not only on the coal or, as is almost invariably the case, the carbonized coal blends, 
but also on the conditions of carbonization (time and temperature) and on the design of the coking plant. All of these factors may play a part in the secondary reactions which occur as the volatile matter passes through the solid or semi plastic coal or the semi-coke layers on its way to the unoccupied space at the top of the coke oven and during its subsequent passage along the ascension pipe ${ }^{29}$. The gas purification applied to remove the hydrogen sulphide and hydrogen cyanide may lead to the production of thiocyanates and ferrocyanides. An alternative procedure is to separate the hydrogen sulphide-containing gases from the absorbent regeneration step and burn these to generate sulphur dioxide and trioxide for the production of sulphuric acid.

Crude benzole is largely a mixture of benzene, toluene and xylenes (i.e., BTX) with a smaller proportion of lower paraffins and naphthalene with traces of carbon disulphide, thiophene and indene ${ }^{23}$. The recovery of this BTX fraction by absorption in an oil has a dual objective: to obtain a valuable saleable commodity and to reduce the amount of BTX in the coke oven gas that is used as fuel. The most important product derived from crude benzole is benzene, the starting point for many chemicals of commercial importance such as nylon, nitrobenzene, dyes, pharmaceuticals and plastics. Toluene is also important as a starting point for chemicals such as trinitrotoluene (TNT) and polyurethane, whilst benzole itself has often been used as motor fuel in the past.

Naphthalene, the most abundant single component in coal tar, is important in the production of phthalic anhydride ${ }^{23}$. Other chemicals obtained from tar include anthracene, which is necessary for the production of anthraquinone dyestuffs, and indene, a component of indene-coumarone resins. The distillation of crude tar also leads to phenol, pyridine, and picolines, all of which are widely used industrial chemicals, and pitch. 


\section{PROCESSING OF COAL COKING BY-PRODUCTS}

\subsection{Tar Distillation}

The aromatic compounds present in tar range from benzene to molecules of several hundreds of $\mathrm{amu}^{23}$.These compounds are mainly polycyclic aromatic hydrocarbons (PAHs), phenolic compounds and oxygenated and nitrogenated aromatic bases and, to a lesser extent, their alkyl derivatives (Figure 2). This makes tar an important source of chemicals (either as single compounds or as mixtures of compounds) for the carbochemical industry.

The main industrial application of tar is as a feedstock for the production of pitch $^{30}$. By means of a distillation process, tar yields a series of liquid fractions with different industrial applications and a solid residue (coal-tar pitch). There are many alternatives for distilling high temperature tar. However, these alternatives basically follow the same sequence of steps (Figure 3). In the first stage, a blend of tars is loaded into a distillation column where BTX (benzene, toluene and xylenes) and water are removed. This fraction contains $<6$ wt. \% of tar, $\sim 4$ wt. $\%$ being BTX. 


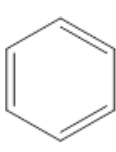

Benzene

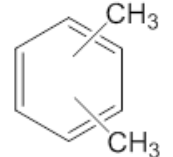

Xylenes<smiles>CC(C)(C)c1ccc2ccccc2c1</smiles>

Methylnaphthalenes

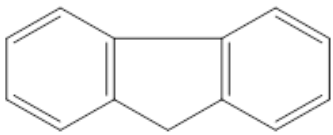

Fluorene

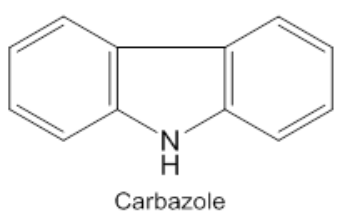

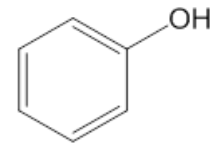

Phenol<smiles>C1=c2ccc3c4c(ccc(c24)=C1)=CC=3</smiles>

Acenaphthylene

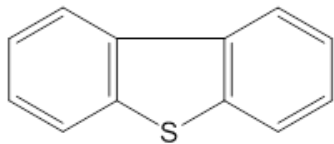

Dibenzothiophene

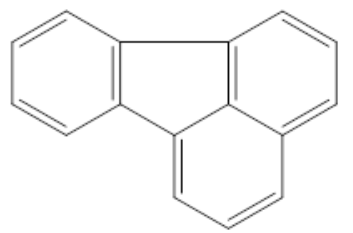

Fluoranthene<smiles>c1ccc2c(c1)C1CC3CC2C1C3</smiles>

Indene<smiles>c1ccc2ncccc2c1</smiles>

Quinoline

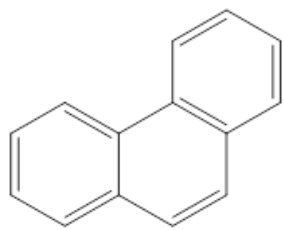

Phenanthrene<smiles>CC1CC2(O)CC3CC1CC2C3</smiles>

Cresols

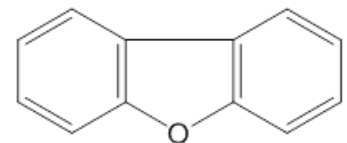

Dibenzofuran

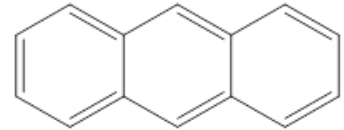

Anthracene

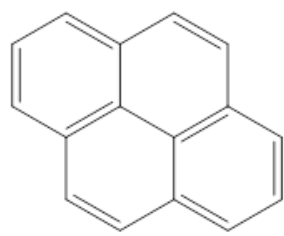

Pyrene

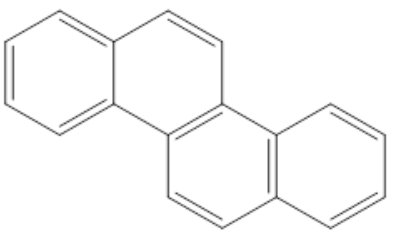

Chrysene

Figure 2. Representative aromatic compounds of a typical coal tar.

Afterwards, the dehydrated tar is made to pass through a second column where it is refined again and carbolic oil, naphthalene oil and wash oil are collected. These fractions constitute around 20 wt.\% of the dehydrated tar. Depending on the distillation process, these three fractions are obtained as a combined fraction, which is subsequently refined again to yield a carbolic oil fraction, that distils at the top of the column $\left(\sim 180-200{ }^{\circ} \mathrm{C}\right)$, a wash oil fraction, which is collected at the bottom of the column $\left(\sim 230-260{ }^{\circ} \mathrm{C}\right)$ and a naphthalene oil fraction, which remains in the middle of the column. Naphthalene oil is the most important fraction from an industrial point of view because it is used for the production of naphthalene, which is the precursor of quite a large number of chemicals. After the carbolic oil, naphthalene oil and wash 


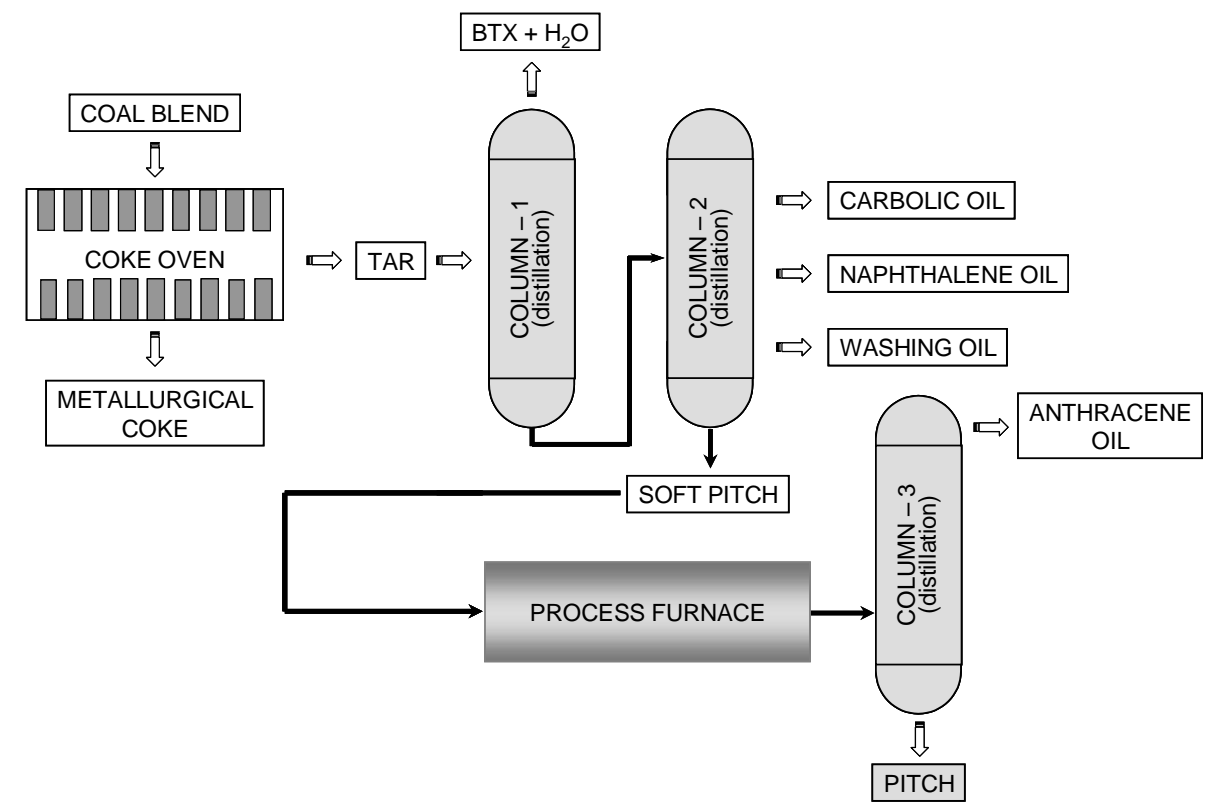

Figure 3. Sequence of steps followed in the distillation of high temperature tar.

oil have been distilled, the fraction left at the bottom of the second column is soft pitch, which in a subsequent step is fed into a reactor where it is heated to temperatures close to $400{ }^{\circ} \mathrm{C}$. Finally, the heated soft pitch is loaded into a third column where the pitch parameters are adjusted (mainly softening point) depending on the application which the pitch is to fulfil (e.g., as binder or impregnation agent). This third distillation stage takes place under adiabatic conditions (with or without vacuum) and besides pitch, yields a fraction called anthracene oil $\left(\sim 260-400{ }^{\circ} \mathrm{C}\right)$, which constitutes approximately $25 \mathrm{wt} \%$ of the dehydrated tar. The global yield of the distillation process is $\sim 50-55$ wt. $\%^{31}$.

\subsection{Coke Oven Gas Treatment}

As described in section 3, the crude gas obtained from the coking of coal in by-product recovery ovens goes through a series of sequential steps to extract the condensable fractions and to remove the hydrogen sulphide and hydrogen cyanide ${ }^{4}$. In general, a gas treatment plant consists 
of primary coolers, followed by retarders, heat exchangers, naphthalene scrubbers, ammonia washers, and benzole scrubbers, but not necessarily in that $\operatorname{order}^{32}$. The cleaned gas is then used to fire the ovens and to generate steam. The composition of the gas is typically $\sim 50$ vol.\% hydrogen with $>25$ vol.\% methane and smaller proportions of carbon monoxide, nitrogen, carbon dioxide and unsaturated hydrocarbons.

\subsection{Other Products}

Coal frequently has associated mineral matter, which is either adventitiously or chemically incorporated into the structure, originated from the mineral constituents of the original plant material from which the coal was first formed and from the seepage of water containing dissolved salts into the decaying plant material ${ }^{33}$. Many of the trace elements such as germanium are present and attempts has been made to recover them, attempts which were not economically feasible at the time. However because of the increasing environmental interest in preventing such elements as mercury from being emitted into the atmosphere, there remains the possibility that in the future the extraction of such elements will become necessary. In this case, they will need to be concentrated by means, for example, by carbonization.

\section{CHEMICALS FROM TAR DISTILLATION FRACTIONS}

Tar is an important source of aromatic chemicals which are used in the synthesis of several products (e.g., resins, polymers, etc.). In order to isolate these chemicals, tar is fractionated by means of a distillation process, which involves gradual heating from room temperature to $\sim 400$ 
${ }^{\circ} \mathrm{C}$. In this way, several fractions are obtained: BTX, carbolic oil, naphthalene oil, wash oil, anthracene oil and coal-tar pitch (the carbonaceous residue obtained after distillation).

\subsection{BTX Fraction}

This fraction is composed of benzene, toluene and xylenes. Its industrial usefulness is becoming less important because of strong competition with other sources such as petroleum derivatives. For this reason, this fraction is usually burned to produce energy for carbochemical installations. Nevertheless, it constitutes a potential source of aromatics that, depending on market conditions, could be used for the production of chemicals. Of the components that make up the BTX fraction, xylenes are the most interesting because they are the raw material (once the three isomers have been separated) used to prepare phthalic anhydride (orto-xylene), isophthalic acid (meta-xylene) and terephthalic acid (para-xylene) ${ }^{34}$ (Scheme 1), which are in turn the basis for the production of various types of industrial monomers and chemicals.

a)

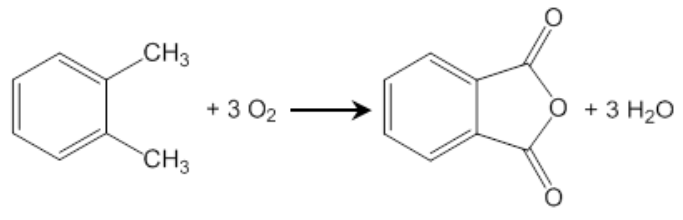

b)

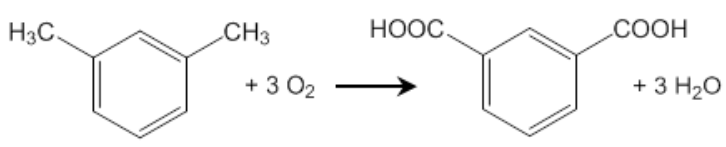

c)

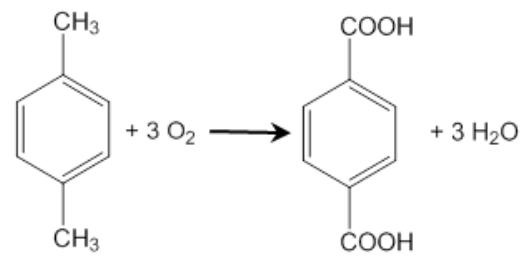

Scheme 1. Synthesis of (a) phthalic anhydride, (b) isophthalic acid and (c) terephthalic acid by oxidation of $o$ - $m$ - and $p$-xylene, respectively. 


\subsection{Carbolic Oil}

The carbolic fraction contains $\sim 25$ wt.\% of phenol and its derivatives (e.g., cresols, xylenols, etc.). A typical composition ${ }^{35}$ of this fraction is shown in Table 1 . The refining of this fraction involves a simple extraction procedure which consists in the use of sodium hydroxide to separate the phenols from the bases and neutral oils and the subsequent neutralization of the salts by applying carbon dioxide to liberate the phenols. Phenol and o-cresol are isolated by distillation, while $\mathrm{m}$ - and $\mathrm{p}$-cresol require more complex processes because of their similar boiling points. Xylenols are usually separated by means of processes that combine distillation and extraction steps $^{36}$. Phenol is the basis for the production of polycarbonates (see section 6.4.1) via the synthesis of bisphenol-A (Scheme 2a), phenolic resins (see section 6.4.2), cyclohexanone/caprolactam (Scheme 2b1/2b2) and alkylphenols. Cresols and xylenols are mainly used in the preparation of resins, herbicides, fungicides, disinfectants, plasticizers, etc. Other minor components of the carbolic fraction are cumene, indane or indene, which are the basis for the production of polymers and flux oil for the production of bitumen.

Table 1. Typical composition of a carbolic oil.

\begin{tabular}{cc}
\hline Family/Compound & Percentage (wt.\%) \\
\hline Phenols & 44 \\
Phenol & 15 \\
o-Cresol & 22 \\
m-Cresol & 11 \\
p-Cresol & 8 \\
Xylenols & \\
\hline Neutral oils & \\
\hline Bases & \\
\hline
\end{tabular}


a)

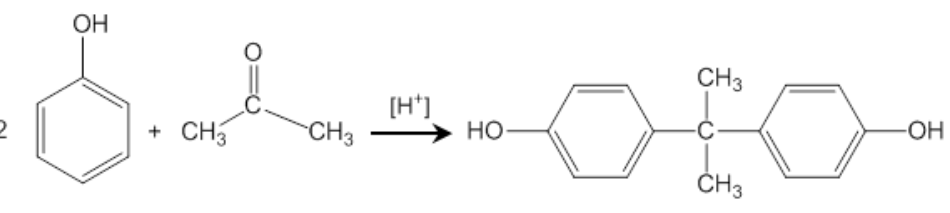

b1)

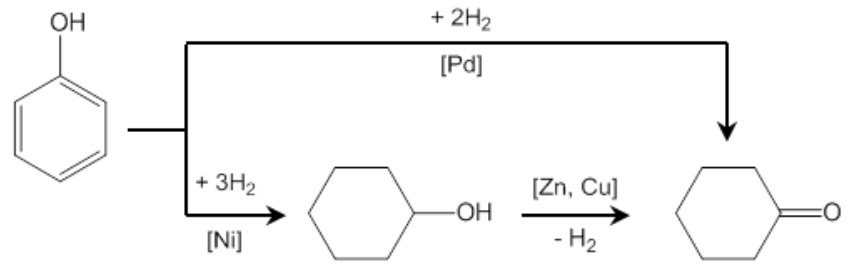

b2)

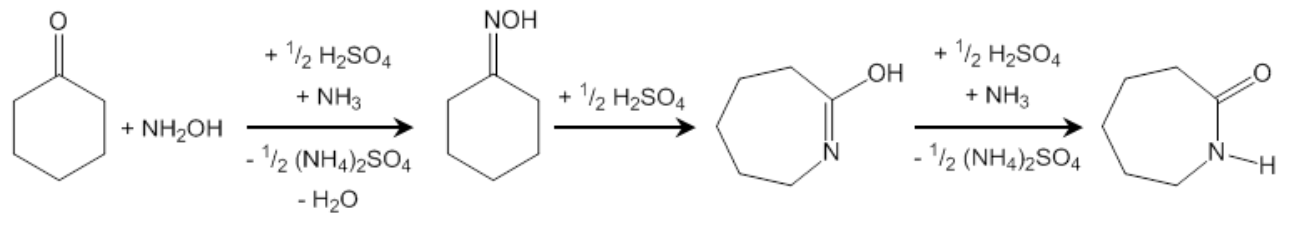

Scheme 2. Synthesis of (a) bisphenol-A and (b1/b2) cyclohexanone/caprolactam from phenol.

\subsection{Naphthalene Oil}

Naphthalene oil is perhaps the coal liquid fraction which is currently generating most economic interest. This is because naphthalene oil is mainly composed of naphthalene ( $60 \mathrm{wt.} \%)$, which is the basis for the synthesis of a large number of chemicals and intermediates that are applied in many industrial sectors (e.g., agriculture, construction, photography, the rubber, tanning and dye industries, polymers, etc. ${ }^{37,38,39,40}$ ), as can be seen in Table 2 . The isolation of naphthalene from the other fraction components is usually performed by means of crystallization, which produces the so-called "technical" and "refined" naphthalenes. The point of crystallization of a typical technical naphthalene is $\sim 78{ }^{\circ} \mathrm{C}$, which corresponds to a naphthalene content of more than 95 wt.\%. This degree of purity can be enhanced to values close to $100 \mathrm{wt} . \%$ by increasing the crystallization point to $\sim 80^{\circ} \mathrm{C}$ (refined naphthalene). 
Table 2. Representative compounds obtained from naphthalene derivatives.

Nitronaphthalene-based compounds

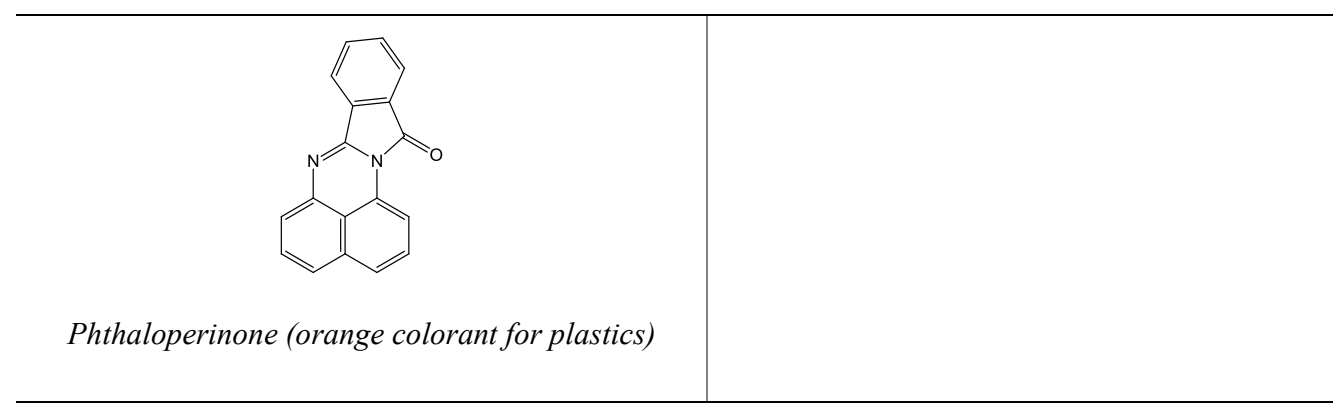

Naphthaleneamine- and naphthalenediamine-based compounds

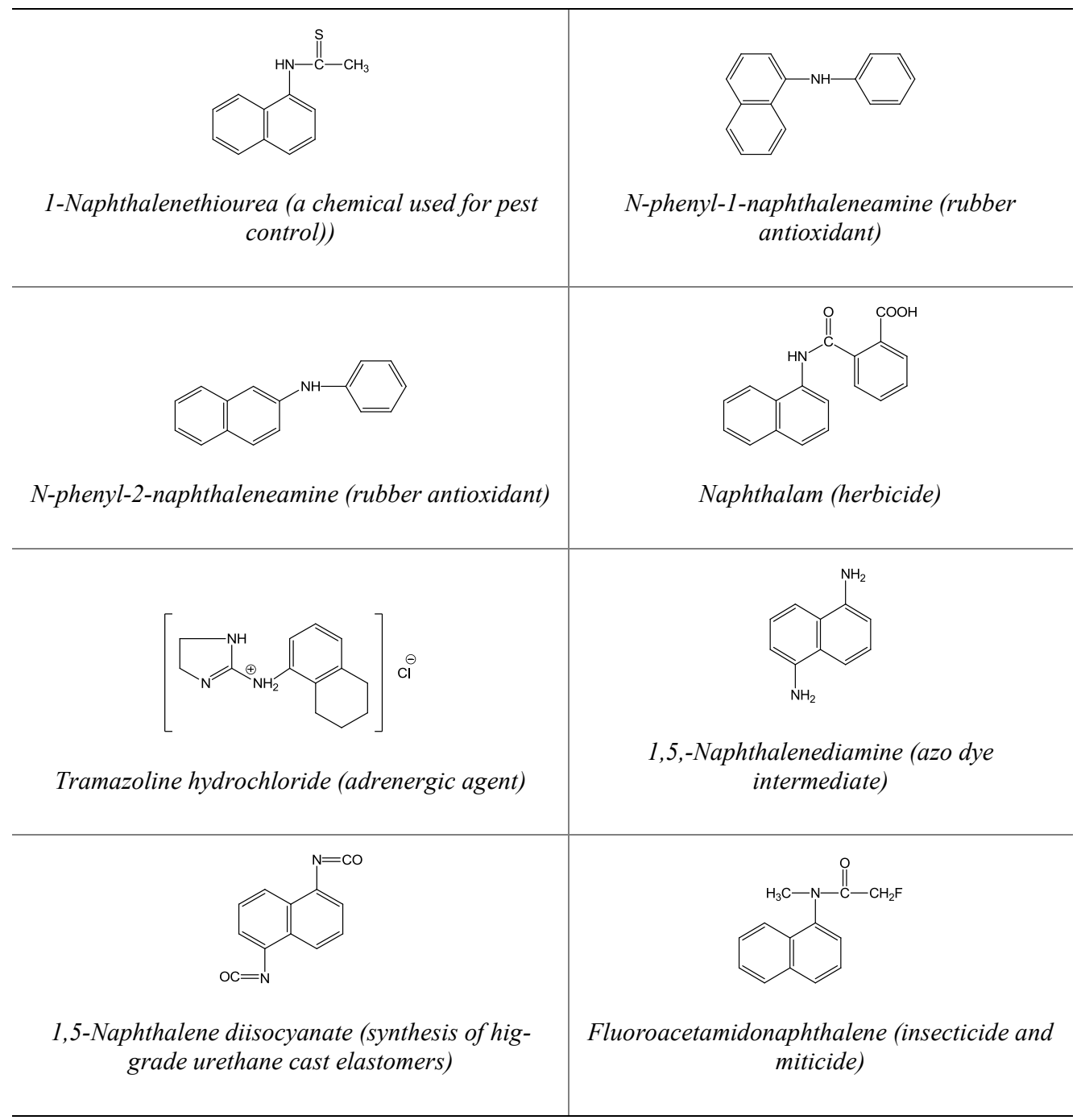

Aminonaphthalenesulfonic acid-based compounds 


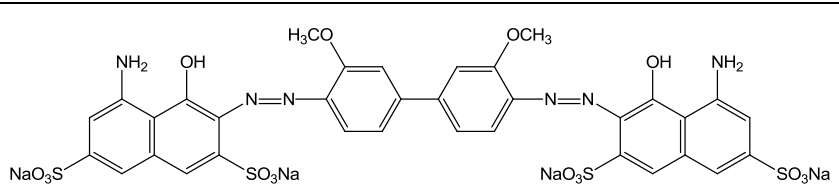

Direct Blue 15 (azo dyestuff)

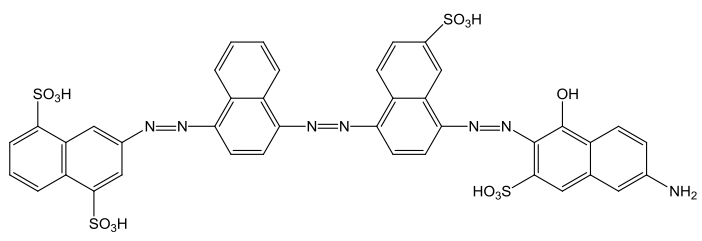

Direct Blue 71 (azo dyestuff)

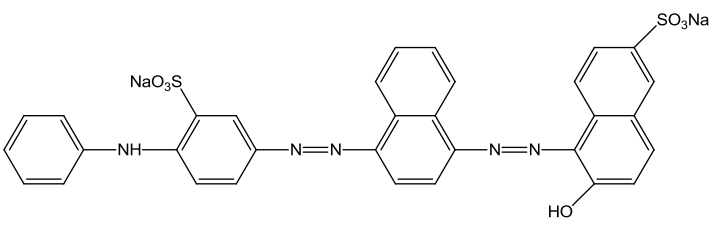

Acid Black 26 (azo dyestuff)

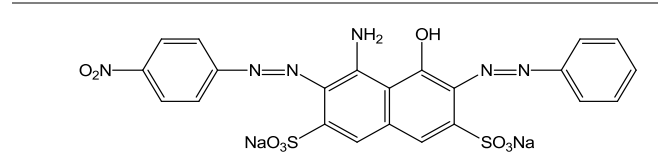

Acid Black 1 (azo dyestuff)

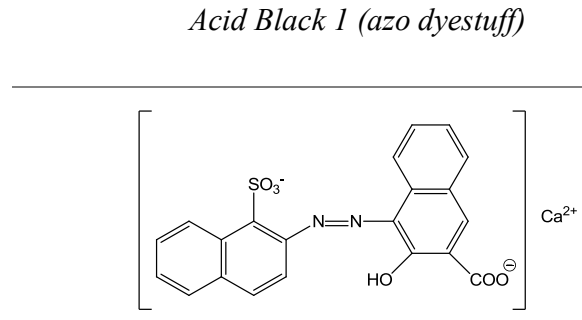

Pigment Red 63:1 (azo dyestuff)

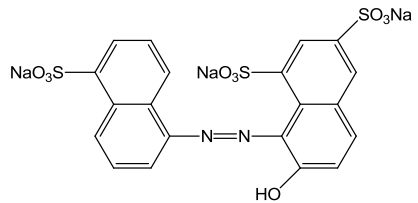

Acid Red 18 (azo dyestuff)

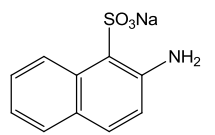

Tobias acid (intermediate pigment)

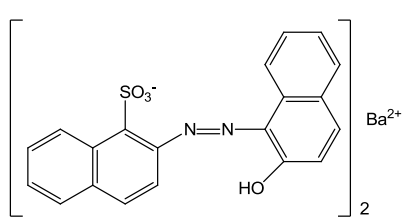

Pigment Red 49:1 (azo dyestuff)

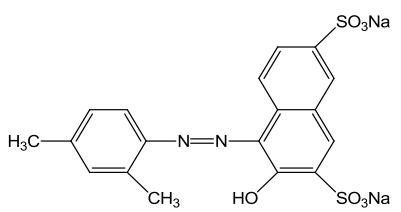

Acid Red 26 (azo dyestuff) 


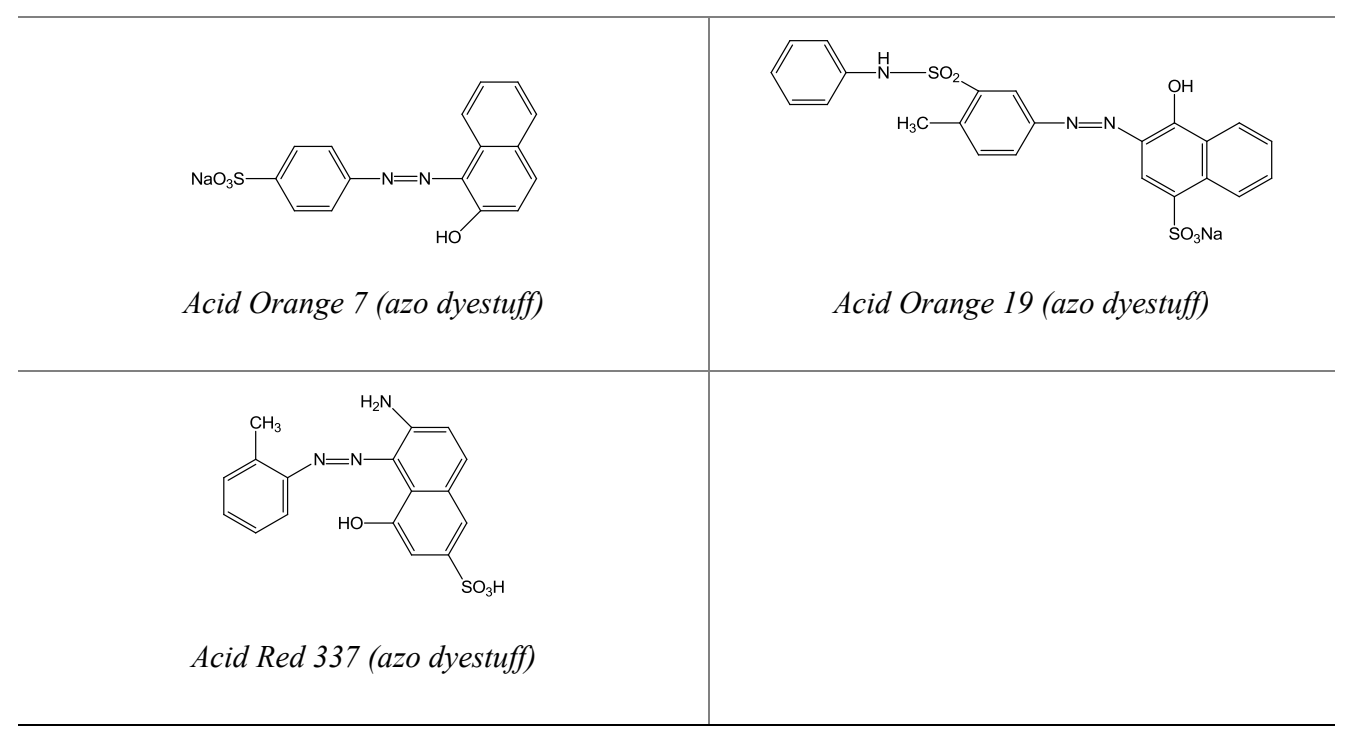

Naphthalenol- and naphthalediol-based compounds

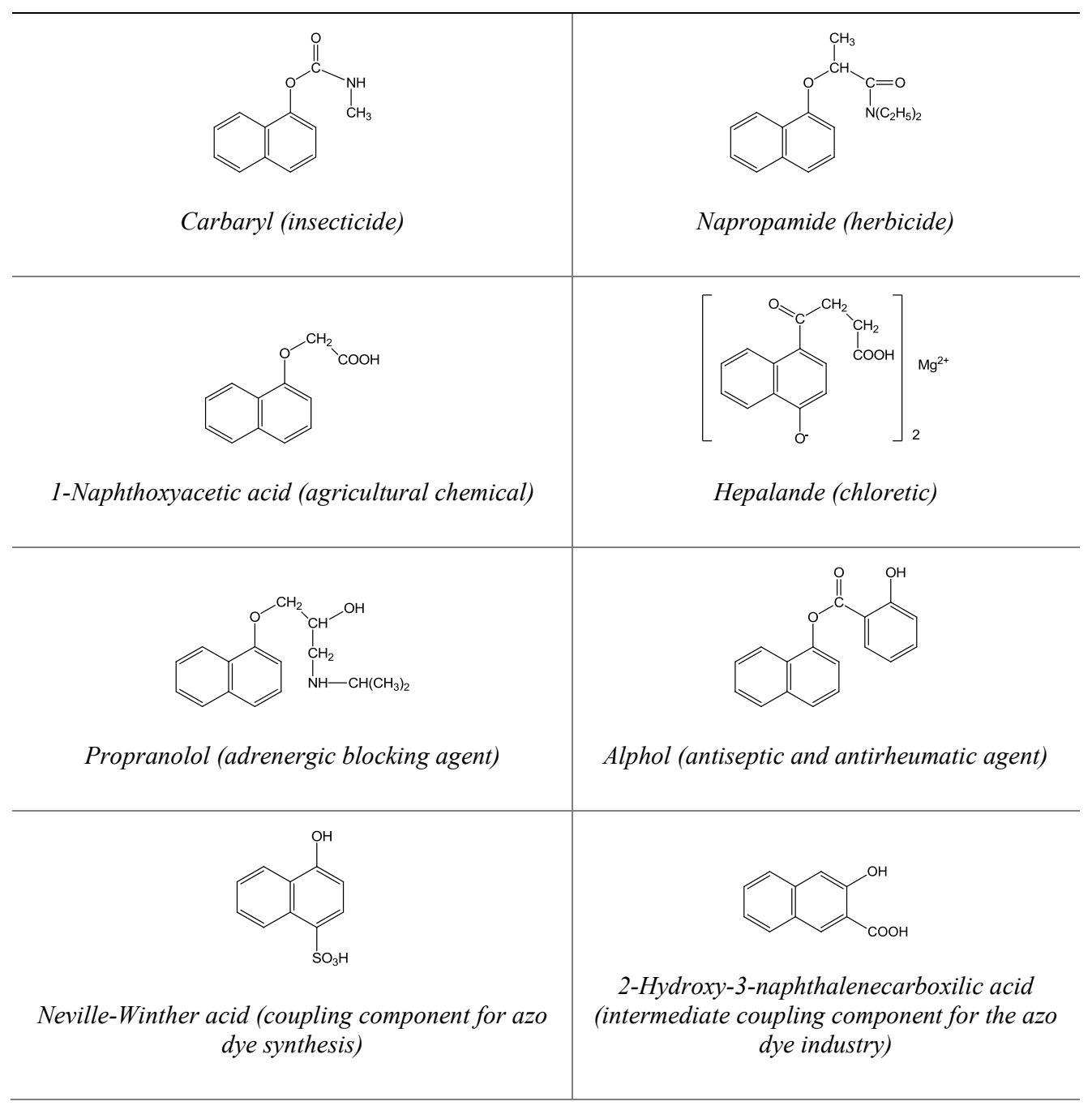




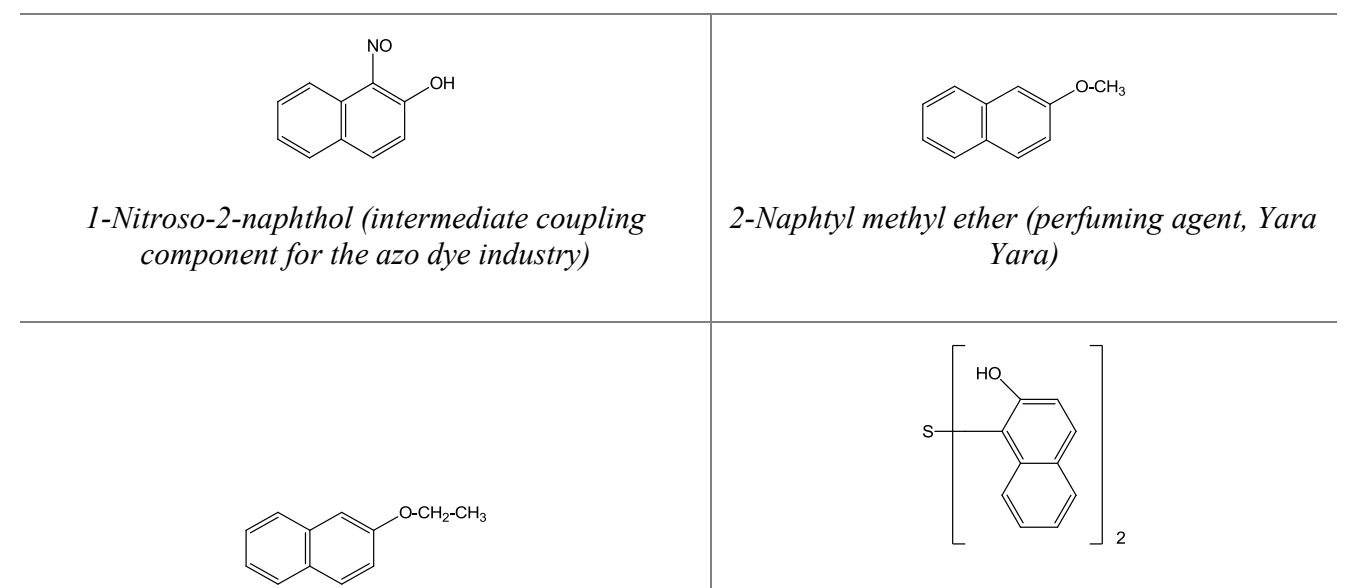

2-Naphtyl ethyl ether (perfuming agent, Bromelia)

Thio-1,1-bis(2-naphthol) (antioxidant for

polyolefins)

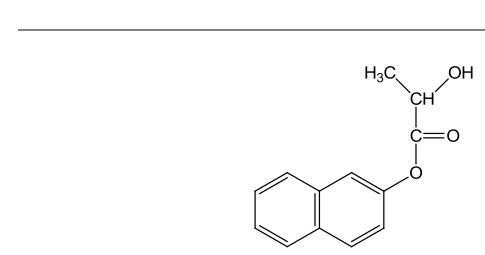

Lactonaphthol (intestinal antiseptic)

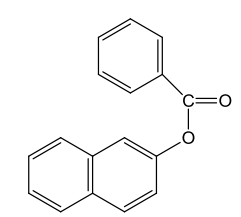

Lintrin (intestinal antiseptic)

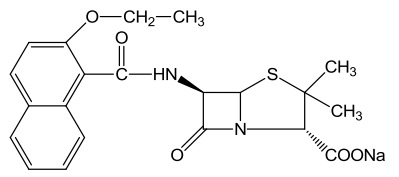

Nafcillin sodium (semisynthetic penicillin)

Tolnaftate (antimycotic and topical antifungal agent)

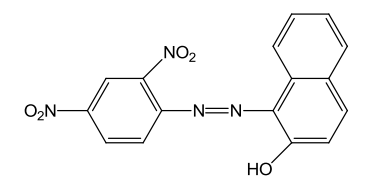

Pigment orange 5 (azo dyestuff)

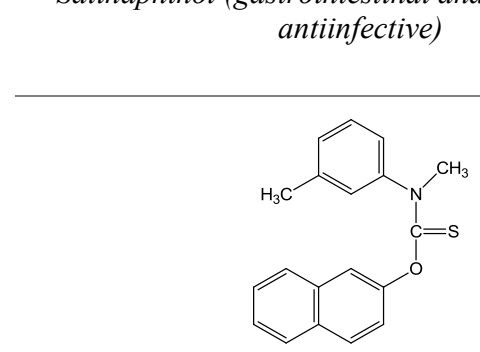

Salinaphthol (gastrointestinal and genitourinary

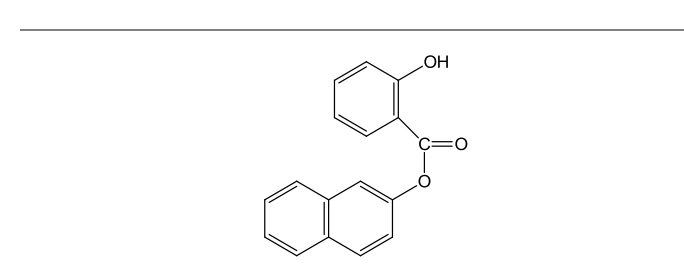




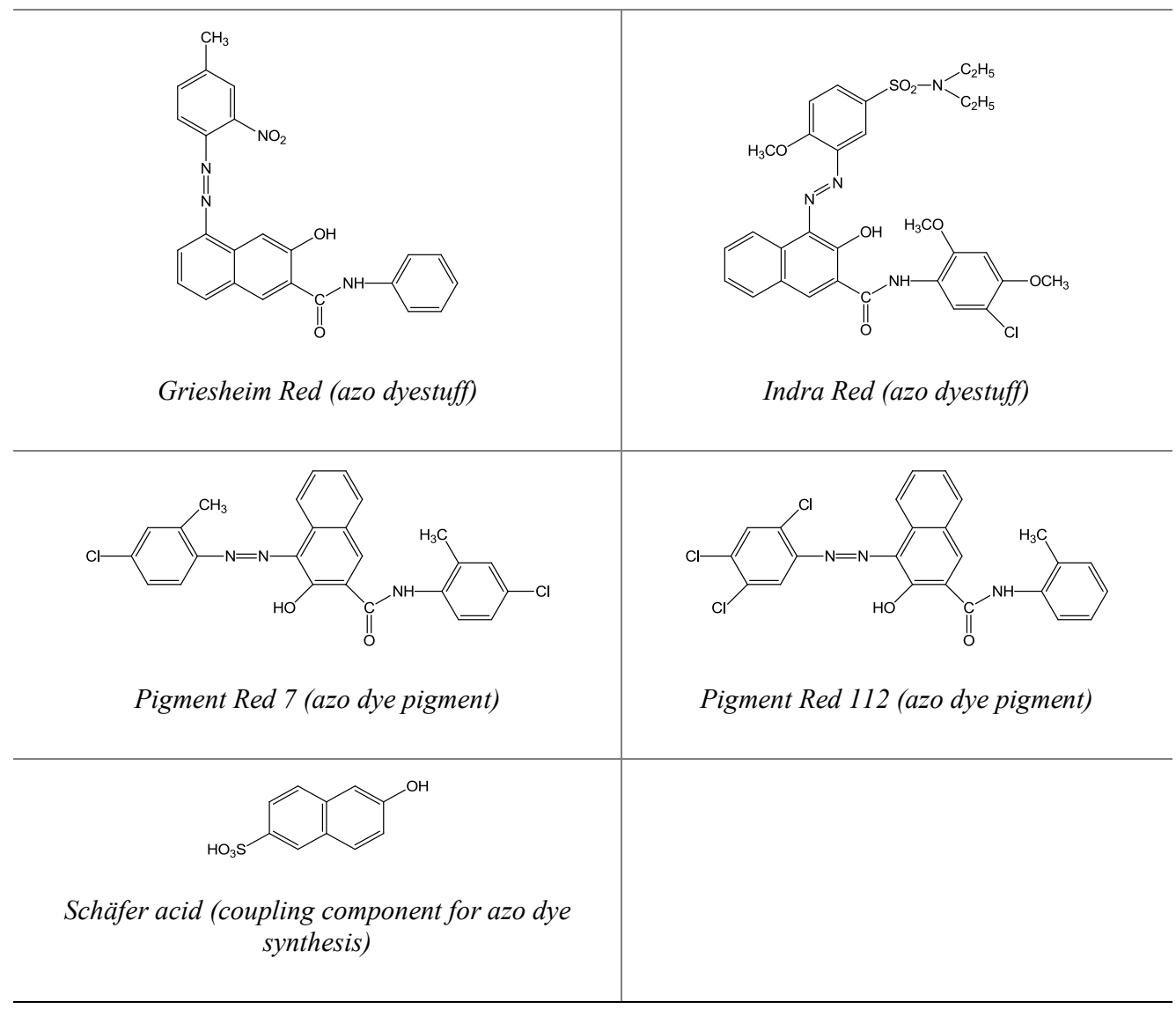

Naphthalenecarboxilic acid- and aminonaphthalenecarboxilic acid-based compounds

Tetrahydrozoline (sympathomimetic and nasal
decongestant agent)
optical bleaches and biological active compounds)
(intermediate for dyes, pigments, optical
bleaches and biological active compounds)

Naphthalenecarboxylic acid-based compounds 


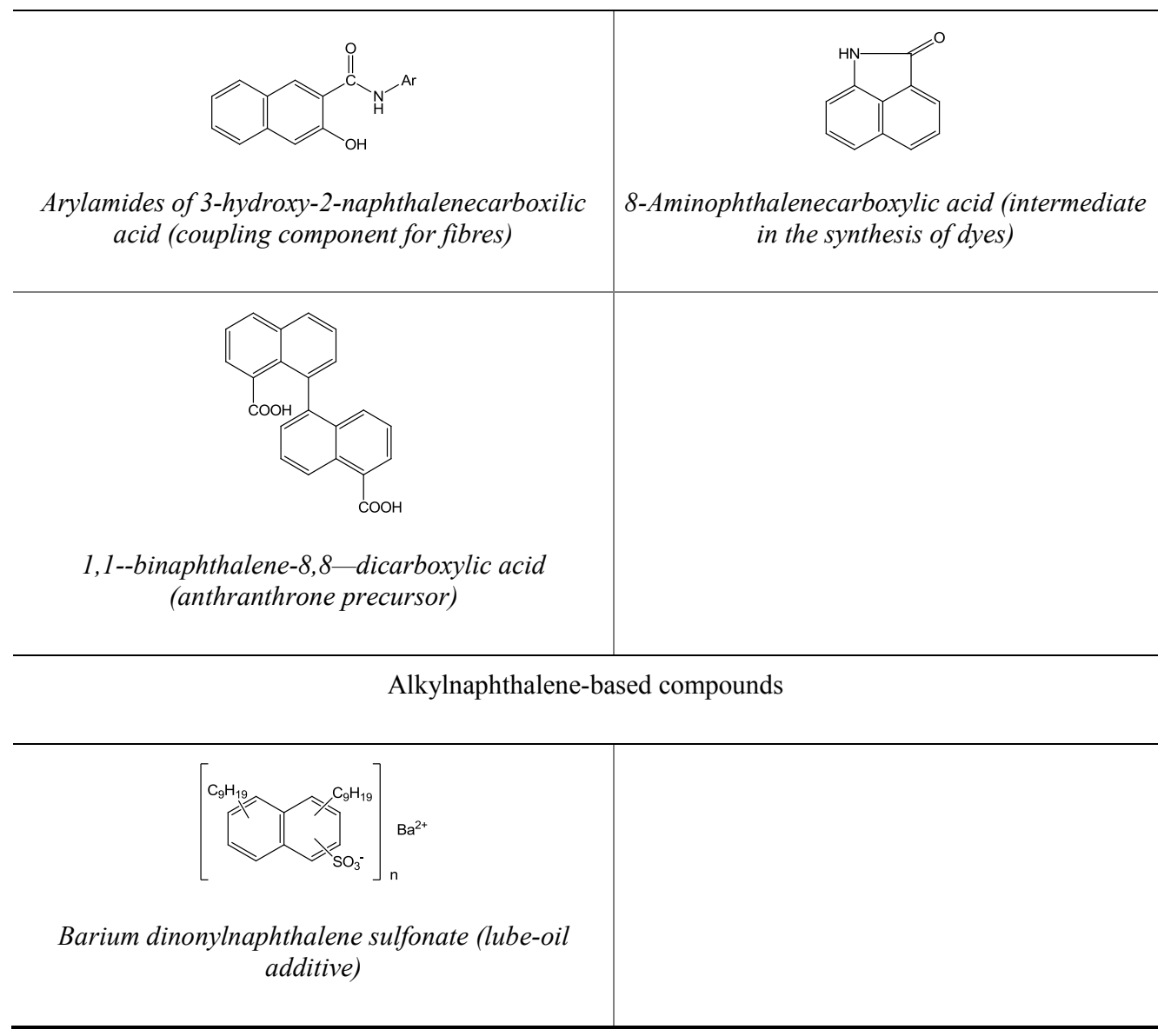

The main application of naphthalene is the synthesis of phthalic anhydride, which is used as an intermediate in the preparation of plasticizers, dyes, resins and specific chemicals (e.g., polyesters, chiral alcohols, etc. $)^{41}$. Phthalic anhydride is obtained from naphthalene by oxidation in the presence of certain catalysts (e.g., $\mathrm{V}_{2} \mathrm{O}_{5}$ supported on silica gel, Scheme 3a). Phthalic anhydride is normally obtained from o-xylene. However, a high yield of phthalic anhydride can be obtained from naphthalene. Other applications of naphthalene include the preparation of: (i) 2-naphthol, either by the alkali fusion of naphthalene-2-sulfonic acid (Scheme 3b1) or by the oxidative cleavage of 2-isopropylnaphthalene (Scheme 3b2), (ii) tetralin and decalin by catalytic hydrogenation processes (Scheme 3c) and (iii) naphthalene sulfonic acids and letter acids by sulfonation/nitration (Scheme $3 \mathrm{~d})^{38} .2$-naphthol is a reference compound in the dye industry and 
a)

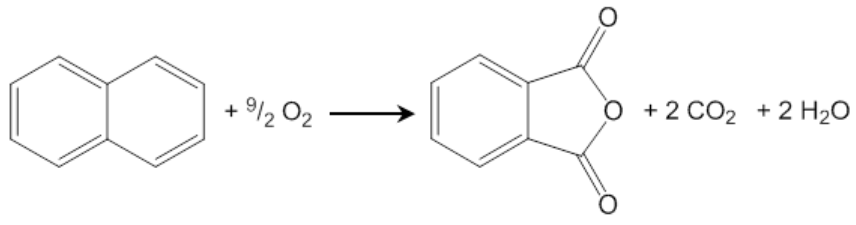

b1)

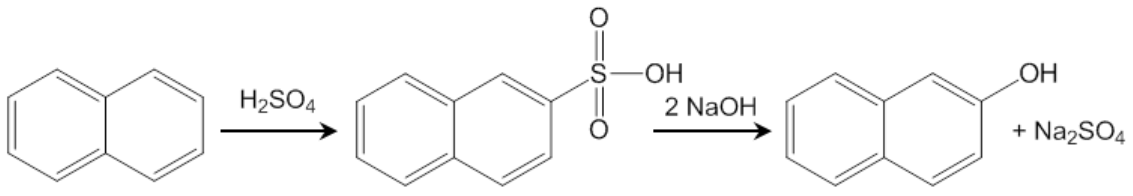

b2)

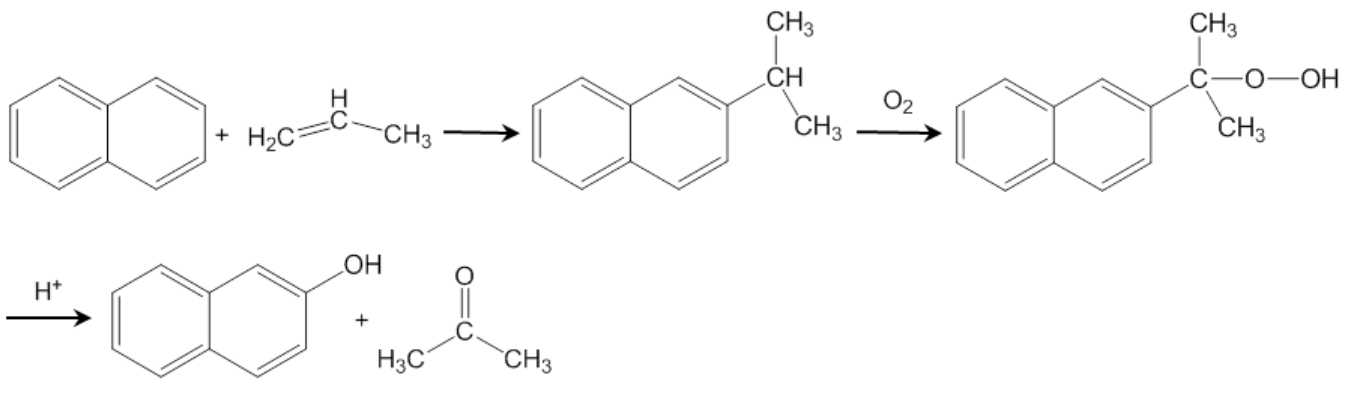

c)

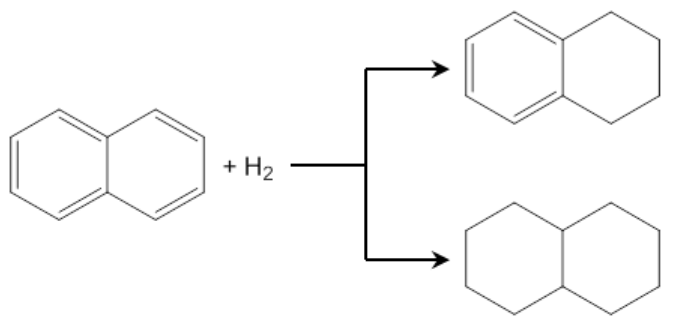

d)

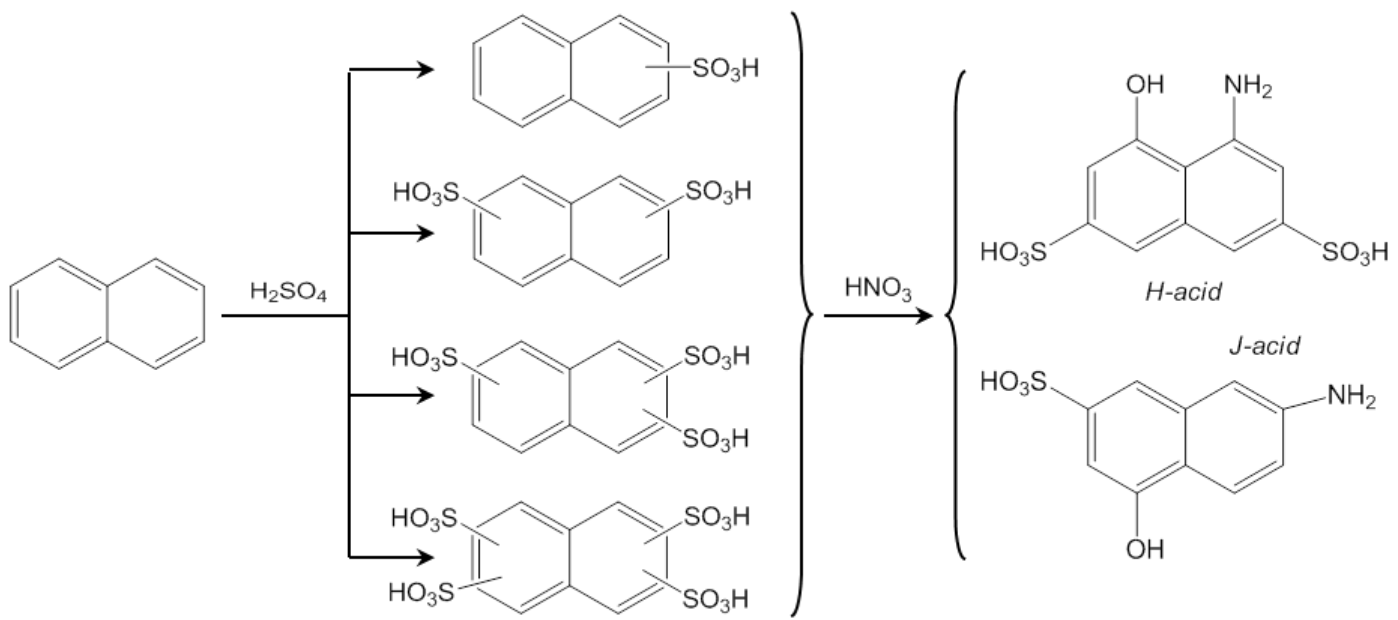

Scheme 3. Synthesis of (a) phthalic anhydride, (b1/b2) 2-naphthol, (c) tetralin/decalin and (d) sulfonic acids / letter acids from naphthalene.

also in the chemical and pharmaceutical sectors where it serves as a precursor for the synthesis of 
several organic compounds (e.g., S-binol, tolnaftate, nafcillin, etc.). Tetralin and decalin are applied as super lubricants in engines that operate at high revolutions (e.g., aircraft). Additionally, tetralin is employed in the production of tetralone (i.e., 3,4-dihydro-2Hnaphthalen-1-one). Sulfonic acids are widely used in the construction sector as fluidizers of concrete, while letter acids (e.g., G-acid, H-acid, J-acid, etc.) are commonly used in the azo-dye industry.

\subsection{Wash Oil}

Nitrogen bases (e.g., quinoline, isoquinoline, carbazol, acridine, etc.) are concentrated in the wash oil fraction. However, these aromatics are not usually isolated and so wash oil is frequently used in the form of a mixture of aromatic compounds. The main characteristics of this fraction are its low freezing point and its excellent behaviour as solvent. For these reasons, wash oil is used in coking batteries to remove impurities from pipes and valves. Additionally, wash oil is also used in blends with anthracene oil as a compensation product for the production of carbon black. The adoption of this strategy will depend on market conditions.

\subsection{Anthracene Oil}

Anthracene oil is the heaviest tar distillation fraction $\left(\sim 260-400{ }^{\circ} \mathrm{C}\right)$. It is composed of compounds of 2-5 aromatic rings. The most representative are acenaphthene, fluorene, phenanthrene, anthracene, fluoranthene and pyrene (Figure 4) ${ }^{42}$. The main application of anthracene oil is to produce carbon black. The conversion of anthracene oil into carbon black involves a gas-phase pyrolysis process at temperatures above $1500{ }^{\circ} \mathrm{C}$ in an air-depleted atmosphere. Under these conditions, the anthracene oil is first vaporized and atomized, giving rise to $\mathrm{C} 1$ and $\mathrm{C} 2$ entities. It is thought that carbon black starts to form via a process of 


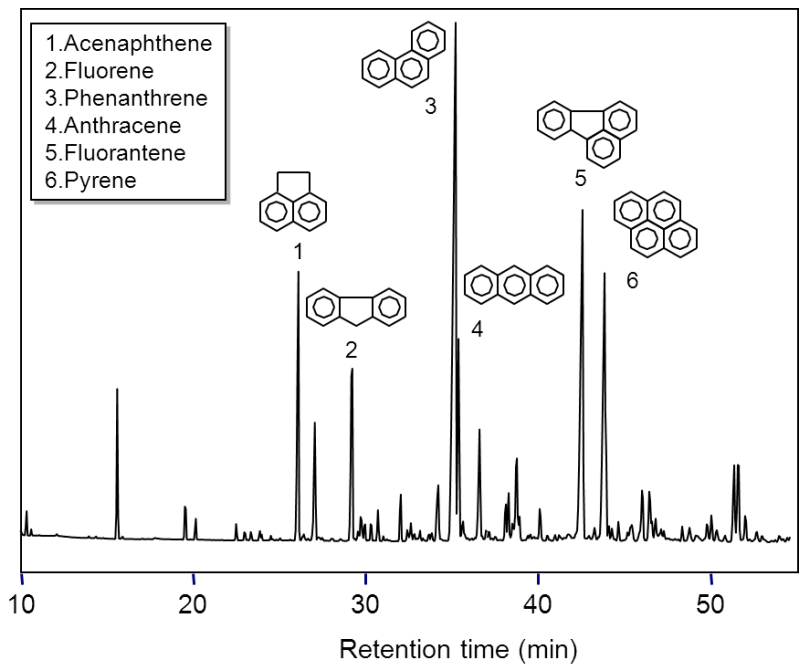

Figure 4. Gas chromatogram of a typical anthracene oil fraction.

nucleation $^{43}$. The process continues via the surface growth and aggregation of particles to finally yield carbon black (Figure 5), which is characterized by its low microstructural order, high porosity and small individual spheres $(<100 \mathrm{~nm})$. Carbon blacks are widely used as a means of reinforcing elastomers (e.g., tyres, rubbers, etc.) and, to a lesser extent, in the pigment industry (e.g., inks, coatings, etc.) and as protection against ultraviolet rays. The production of carbon black from anthracene oil frequently faces the problem of a fluctuating market clearly dominated by petroleum derivatives. As a result, anthracene oil is sometimes considered as a mere residual product that is difficult to eliminate and, at other times, the most valuable fraction of tar

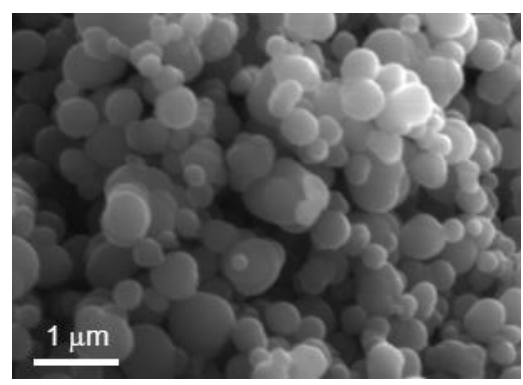

Figure 5. SEM image of carbon black. 
distillation, even more so than the coal-tar pitch.

Another application of anthracene oil is as a source of anthracene ${ }^{44}$. While naphthalene constitutes $\sim 60 \mathrm{wt} . \%$ of naphthalene oil, anthracene only represents $\sim 5 \mathrm{wt} \%$ of anthracene oil, which makes it more difficult to isolate from the other components of the fraction. Despite this, anthracene oil is currently an important source of anthracene. The separation of anthracene is carried out by a crystallization process in which anthracene is concentrated up to values of $\sim 25$ 30 wt.\%. After vacuum distillation, this value is increased to $~ 50 \mathrm{wt} . \%$. Pure anthracene $(\sim 95$ wt.\%) is obtained by a process of re-crystallization in polar solvents.

One of the main uses of anthracene is the synthesis of anthraquinone. Anthraquinone can be produced from anthracene (Scheme $4 \mathrm{a}$ ) by either a liquid-phase oxidation process with $\mathrm{CrO}_{3}$ at moderate temperatures $\left(50-100{ }^{\circ} \mathrm{C}\right)$ or by a gas-phase oxidation process at temperatures close to $400{ }^{\circ} \mathrm{C}$, using $\mathrm{FeVO}_{4}$ as catalyst. An alternative route for the preparation of anthraquinone from coal-based chemicals is based on the acyclation of benzene with phthalic anhydride ${ }^{45}$ (Scheme $4 b)$.

In addition to anthracene, anthracene oil is rich in other PAHs, such fluorene, phenanthrene, fluoranthene, pyrene, etc. These compounds have until now attracted little interest because their industrial application is in specific fields (e.g., herbicides, fluorescent dyes, etc.). However, it is worth noting that the only source of this family of PAHs is anthracene oil. These PAHs can be isolated from anthracene oil by means of complex techniques that usually involve distillation, filtration, crystallization, etc. They can then be transformed into ketones and other intermediates for the synthesis of several chemicals (e.g., fluorenone, 9,10-phenanthrenequinone, perinone pigments, etc. $)^{10}$. 
a)

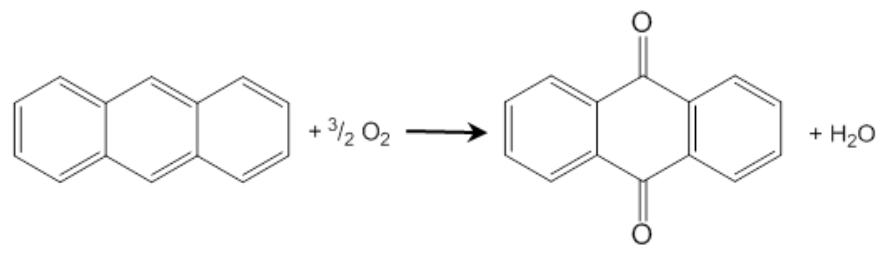

b)

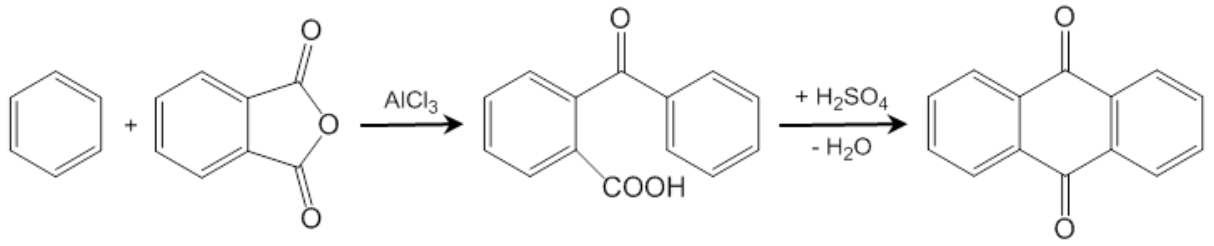

Scheme 4. Synthesis of anthraquinone (a) by oxidation of anthracene and (b) by acyclation of benzene with phthalic anhydride.

\subsection{Coal-Tar Pitch}

Coal-tar pitch is the residue left by the tar after distillation. Consequently, coal-tar pitch concentrates the heaviest components of the tar. It is used in the aluminium and steel industry as binder and impregnating agent for the production of carbon anodes and graphite electrodes ${ }^{9,30,31}$. In addition to these traditional applications, it is also employed for the production of graphitizable carbons $^{46}$ (i.e., materials able to retain a graphitic structure after being subjected to treatment above $2500^{\circ} \mathrm{C}$ ).

\section{POLYMERS FROM COAL COKING CHEMICALS}

As previously mentioned, aromatics from coal coking are used in several fields related to the chemical, pharmaceutical or dye industries. Another important field of application is as intermediates in the production of polymers. The use of aromatics from coal coking products in this field is limited by the strong competition with aromatics from crude oil. 
Among the aromatics obtained from coal coking, xylene, naphthalene and phenol derivatives are those that offer the greatest possibilities, especially in the production of polymers and resins. The preparation of monomers from xylene and naphthalene usually requires some preliminary reactions in order to obtain the xylene or naphthalene derivative, which will be used as a precursor of the monomers.

The following section, apart from describing the main synthesis routes, deals with the specific applications of each material.

\subsection{Xylene and Naphthalene Derivatives as Intermediates in the Production of Polymers}

The xylene derivatives most commonly used to produce polymers are terephthalic acid (Scheme 1), terephthaloyl dichloride, isophthaloyl dichloride and dimethylterephthalate. Terephthaloyl dichloride is synthesized by causing terephthalic acid to react with phosphorus pentachloride ${ }^{47}$ (Scheme 5a). Isophthaloyl dichloride, which is commonly used in the synthesis of polyaramids, is industrially produced by the chlorination $\left(\mathrm{SOCl}_{2} / \mathrm{Cl}_{2}\right)$ of m-xylene via 1,3-bis-trichloromethylbenzene and the subsequent reaction of this intermediate with isophthalic acid ${ }^{48}$ (Scheme $5 b$ ). Dimethylterephthalate can be produced by various routes, but the one based on the oxidation/esterification of p-xylene is the most widely used ${ }^{49,50}$. This route involves the catalytic oxidation of $\mathrm{p}$-xylene with cobalt and manganese derivatives to produce $\mathrm{p}$-methylbenzoic acid. After that, esterification of the acid with methanol gives rise to a p-methylester. Subsequent oxidation leads to a terephthalic acid monomethylester, which after esterification gives rise to dimethylterephthalate (Scheme 5c).

One of the most representative naphthalene derivatives for the production of polymers is dimethyl-2,6-naphthalenedicarboxilate, which is obtained from 2,6-dimethylnaphthalene. The 
a)<smiles>O=C(O)c1ccc(C(=O)O)cc1</smiles>

b)<smiles>Cc1cccc(CCCCCCC(C)(Cl)Cl)c1</smiles><smiles>O=C(O)c1cccc(C(=O)OCCCCC(=O)c2cccc(C(Cl)(Cl)Cl)c2)c1</smiles>

c)

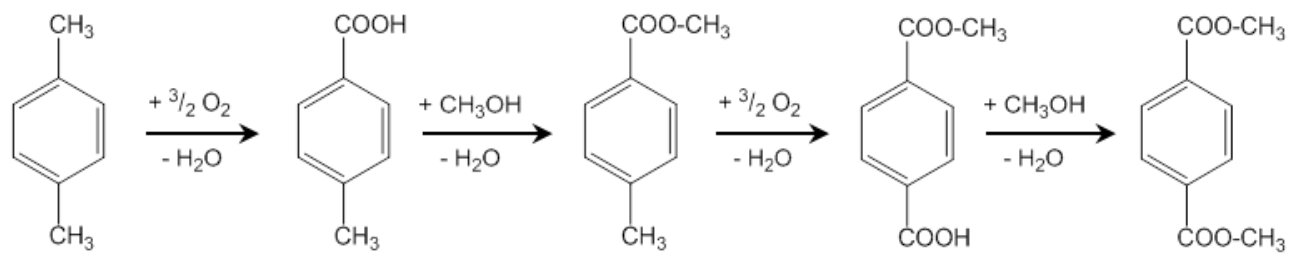

Scheme 5. Synthesis of (a) terephthaloyl dichloride, (b) isophthaloyl dichloride and (c) dimethylterephthalate from terephthalic acid, m-xylene and p-xylene, respectively.

latter is usually produced from o-xylene and butadiene by means of a condensation process, which involves four subsequent reaction steps $^{51}$ (Scheme 6a). Initially, o-xylene reacts with butadiene in the presence of a basic liquid catalyst $(\mathrm{Na} / \mathrm{K})$ to give 5-o-tolylpentene. In a second step, 5-o-tolylpentene is cycled to form 1,5-dimethyltetraline, which in a subsequent reaction is transformed into 1,5-dimethylnaphthalene by dehydrogenation. Finally, 1,5-dimethylnaphthalene is isomerized to 2,6-dimethylnaphthalene. The oxidation of 2,6-dimethylnaphthalene with air in the presence of $\mathrm{Co} / \mathrm{Br}$-catalysts gives rise to 2,6-naphthalenedicarboxilic acid, which, after esterification with methanol leads to dimethyl-2,6-naphthalenedicarboxilate ${ }^{52}$, Scheme 6 b.

\subsection{Xylene-Based Polymers}

The main xylene-based polymers are polyesters, aramids and benzobisoxazoles. 
a)

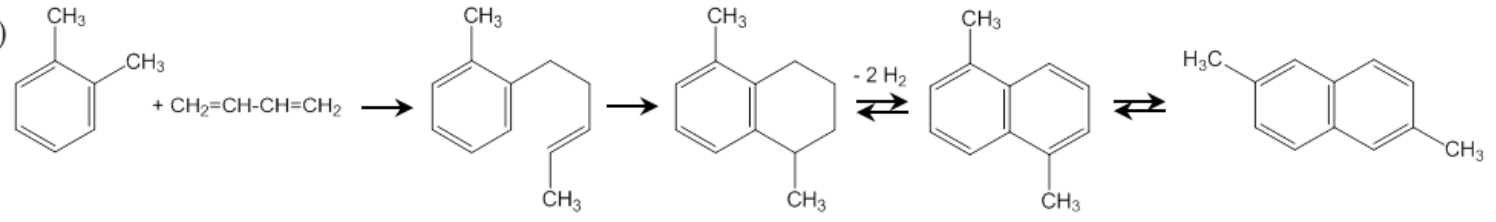

b)

Scheme 6. Synthesis of (a) 2,6-dimethylnaphthalene and (b) dimethyl-2,6napthalenecarboxilate from o-xylene.

\subsubsection{Polyesters}

The most common xylene-based polyesters are poly(ethylene terephthalate), poly(trimethylene terephthalate) and poly(butylene terephthalate).

Poly(ethylene terephthalate), usually known as PET, is the third most commonly produced polymer after polyethylene and polypropylene. PET is produced in two ways ${ }^{53}$ : (i) by esterification of terephthalic acid and ethylene glycol at moderate temperatures $\left(\sim 220-260^{\circ} \mathrm{C}\right)$ and pressures ( 2-6 bar) to produce the polymer and water as a by-product (Scheme 7a1) and (ii) by means of ester interchange of dimethylterephthalate with an excess of ethylene glycol at moderate temperature $\left(\sim 150-200^{\circ} \mathrm{C}\right)$ in the presence of a basic catalyst such as antimony trioxide (Scheme 7a2). The second route, gives rise to methanol as a by-product. This methanol is removed by distillation to favour the reaction. Any excess of ethylene glycol is also removed by vacuum distillation. Polycondensation takes place in a second step at higher temperatures $\left(\sim 275^{\circ} \mathrm{C}\right)$ during the continuous distillation of ethylene glycol. PET may undergo degradation during the processing (e.g., thermal oxidation). In order to mitigate this effect, co-monomers that reduce the melting point of the polymer are used (e.g., cyclohexane dimethanol). PET is mainly used to produce fibres and plastic containers, such as bottles. 
a1)

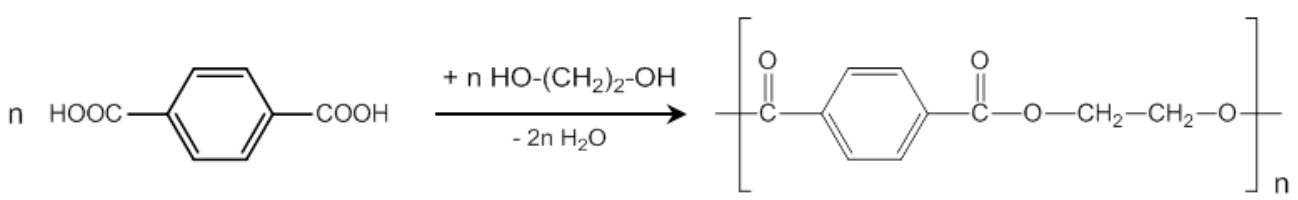

a2)
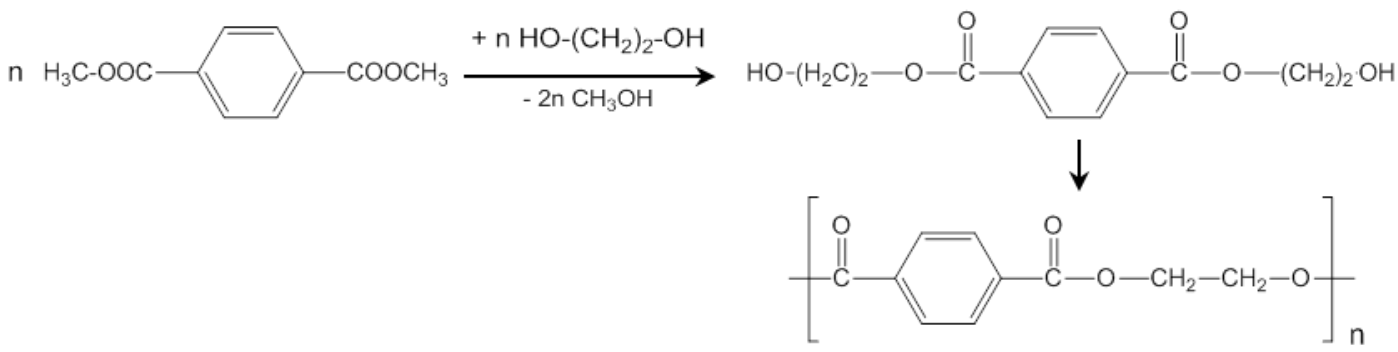

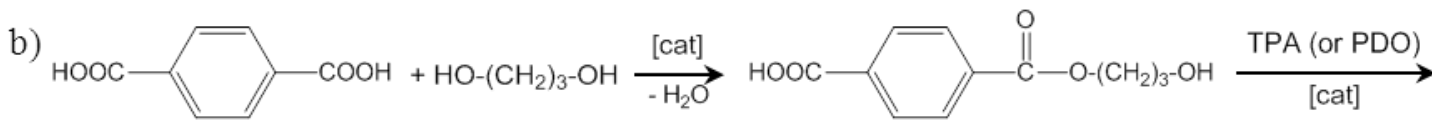

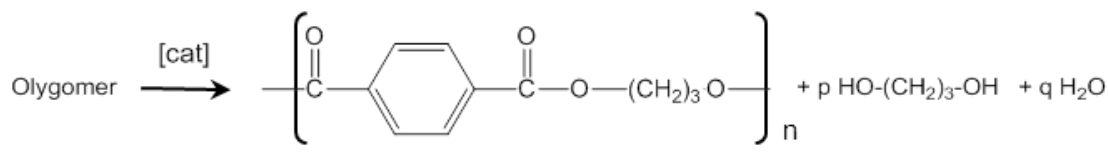

c1)

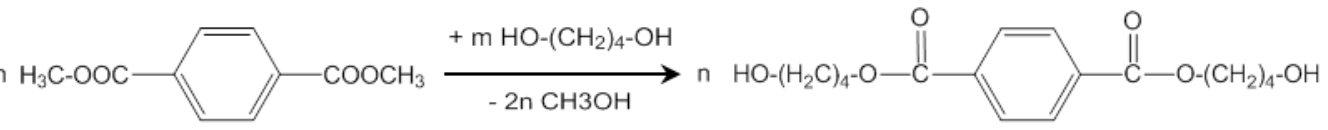

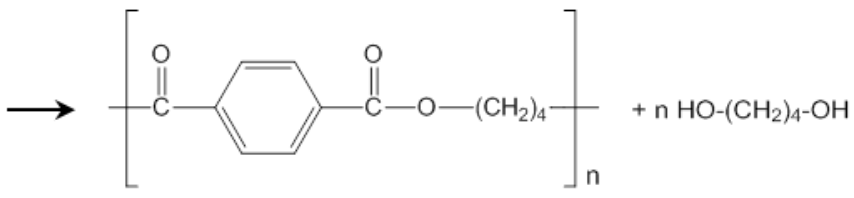
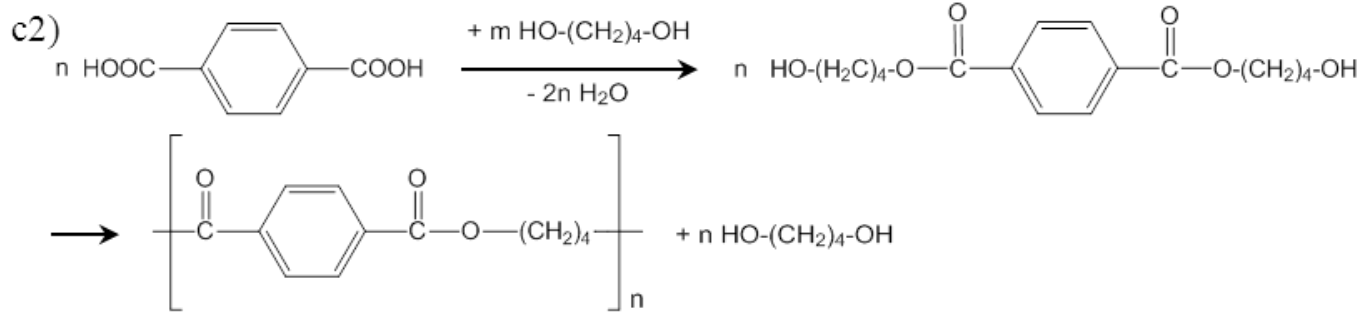

Scheme 7. Synthesis of xylene-based polymers: (a) poly(ethylene terephthalate), (b) poly(trimethylene terephthalate) and (c) poly(butylene terephthalate).

Poly(trimethylene terephthalate) is an aromatic polyester obtained by the reaction of terephthalic acid with 1,3-propanediol ${ }^{54,55,56}$. The conventional route of synthesis requires the use of high 
temperature, high-vacuum systems and the presence of a catalyst to favour the reaction. Of the catalysts tested, those containing titanium seem to be the most appropriate (e.g., titanium dioxide). The synthesis occurs by means of an esterification process that gives rise to the monomer and then the chain grows by esterification with terephthalic acid and/or 1,3,propanediol to produce the oligomers as water is removed (Scheme 7b). Poly(trimethylene terephthalate) is a recognized material that is mainly used in the production of isotropic fibres which are soft and, at the same time, stain resistant. DuPont and Shell are commercializing poly(trimethylene terephthalate)-based fibres (Sonora and Corterra fibres, respectively) for the manufacture of clothing, automobile fabrics, etc.

Poly(butylene terephthalate) can be prepared by means of two different procedures ${ }^{53,57}$ : (i) transesterification of dimethyl terephthalate in the presence of an excess of 1,4-butanediol and subsequent polycondensation of the resulting bis-hydroxybutylterephthalate (Scheme 7c1). This procedure can be performed step by step (batch process) or in continuous mode, (ii) using terephthalic acid instead of dimethyl terephthalate. In this procedure, water is distilled in order to shift the esterification equilibrium towards the final product (Scheme 7c2). The catalysts most frequently used for these procedures are titanium-based. Although the second procedure is cheaper than the first, the dimethyl terephthalate-based process is still used in most industrial plants. This is because the main by-product formed during these processes (i.e., tetrahydrofuran THF) is highly contaminated and it is produced in larger amounts in the terephthalic acid-based process $^{58}$. Poly(butylene terephthalate) is a semi-crystalline thermoplastic polyester that is classified as a medium performance engineering polymer. It provides a valuable combination of technical properties (e.g., resistance to heat and creep and good chemical processability, etc.) that makes it a suitable material for a variety of applications. Poly(butylene terephthalate) is currently 
used in the automobile, electrical and electronic sectors where it is employed as a substitute for metals and thermosetting resins.

\subsubsection{Aramids}

Aramids are usually obtained by a combination of an aromatic diamine and an an aromatic carboxylic acid dihalide. Among the aramid polymers, poly(p-phenylene terephthalamide) and poly(m-phenylene isophthalamide) are perhaps the most commonly used for the production of carbon fibres.

Poly(p-phenylene terephthalamide) is probably the most widely known aramid polymer because it is used for the production of Kevlar and Twaron fibres ${ }^{59}$. Poly(p-phenylene terephthalamide) is prepared from p-phenylenediamine and terephthaloyl dichloride with the formation of hydrochloric acid as by-product ${ }^{60}$ (Scheme $8 \mathrm{a}$ ). Hexamethylphosphoroamide was used as solvent initially but this solvent has been replaced for safety reasons by a solution of N-methyl-2pyrrolidinone and calcium chloride. The fibres obtained from these polymers exhibit extraordinarily high strength, one of their most popular applications being the manufacture of bullet proof vests.

Poly(m-phenylene isophthalamide) is a polymer that is commercialized under the name of Nomex (DuPont). This polymer is synthetized from the monomers, m-phenylenediamine and isophthaloyl dichloride. Hydrogen chloride is removed as a by-product ${ }^{59,61,62}$ (Scheme $8 b$ ). The meta arrangement of the bonds in the polymer makes their alignment during the formation of the filaments impossible. As a result, these fibres do not exhibit mechanical properties as good as the Kevlar fibres (para arrangement of the bonds). However, they show an excellent thermal 
a)<smiles>CCOc1ccc(C(=O)Nc2ccc(NC(C)C)cc2)cc1</smiles>

b)<smiles></smiles>

c)<smiles>Cc1ccc(-c2nc3cc4nc(C(C(=O)O)C(C)(C)C)oc4cc3o2)cc1</smiles>

Scheme 8. Synthesis of xylene-based polymers: (a) poly(p-phenylene terephthalamide) (b) poly(m-phenylene isophthalamide) and (c) poly(p-phenylene-2,6-benzobisoxazole).

behaviour, which makes them suitable for applications such as flame retardant material (e.g., firefighting equipment).

\subsubsection{Benzobisoxazoles}

Benzobisoxazole is the basis for the production of a family of polymers widely used in the production of fibres (Zylon fibres, Toyobo Co.). These fibres are produced from poly(pphenylene-2,6-benzobisoxazole), commonly known as PBO. The most important method for the synthesis of PBOs is the high temperature polycondensation $\left(130-200{ }^{\circ} \mathrm{C}\right)$ of bis(oaminophenol)s with aromatic diacids or their derivatives in polyphosphoric acid (PPA) ${ }^{63}$ (Scheme 8c). PPA acts as condensing agent and solvent. Several methods of preparing PBOs 
have been developed. These employ one-step and two-step processes from the combination of bis(o-aminophenol) derivatives and aromatic dicarboxylic acid analogues ${ }^{64,65}$. Due to its molecular structure, PBO shows excellent mechanical, thermal and chemical behaviour, which makes it a suitable material for use in high performance applications. However, its range of applications is restricted by its poor solubility, the production of fibres being its main application (Zylon fibres). These fibres exhibit even better mechanical properties than Kevlar fibres.

\subsection{Naphthalene-Based Polymers}

Naphthalene and its derivatives are reference compounds for the synthesis of a wide variety of polymers. 2,6-dimethylnaphthalene carboxilate is a versatile monomer that can be used to produce polymers that exhibit physical and mechanical properties generally superior to those of polymers made from terephthalic acid or dimethylterephthalate ${ }^{66}$. This is because the incorporation of a double-ring structure in the polymer chain increases thermal, chemical, mechanical and barrier performance with respect to polymers based on a single aromatic ring. These polymers, despite their high manufacturing cost, are being introduced onto the market as competitive polymers so successfully that they are even replacing single-ring polymers in many applications. The most prominent of these naphthalene-based polymers are poly(ethylene naphthalate), poly(trimethylene naphthalate) and poly(butylene naphthalate). The production of these polymers is carried out by similar routes of synthesis: esterification of 2,6dimethylnaphthalene carboxilate with the corresponding diol (i.e., ethylene glycol and propylene glycol and butylene glycol, respectively) in the presence of catalysts ${ }^{67}$ (Scheme 9).

Poly(trimethylene naphthalate) and poly(butylene naphthalate) are relatively new polymers that can be expected to be used as precursors of fibres and resins ${ }^{68}$. More interesting is poly(ethylene 
a)

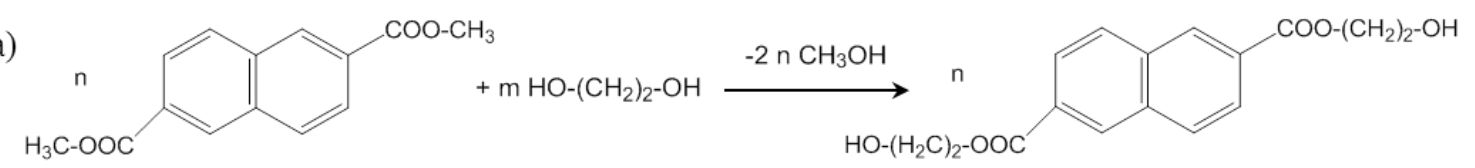
$\longrightarrow-\mathrm{OC}$

b)

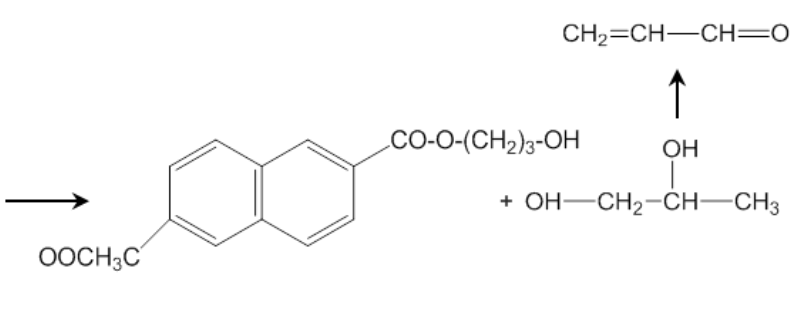
(DNC) (PDO)

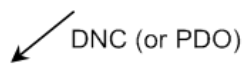<smiles>COC(=O)c1ccc2cc(C(=O)OC[As](C)(C)OCC(C)O)ccc2c1</smiles>

c)

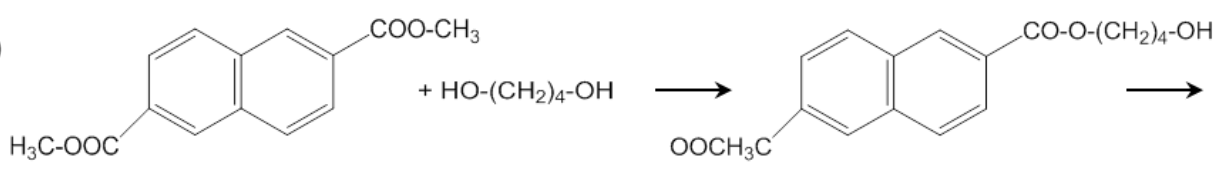<smiles>COCCCCCCOC(=O)c1ccc2cc(C(=O)OC)ccc2c1</smiles>

Scheme 9. Synthesis of naphthalene-based polymers: (a) poly(ethylene naphthalate) (b) poly(trimethylene naphthalate) and (c) poly(butylene naphthalate).

naphthalate), which is employed in the manufacture of fibres, films, special containers, etc. The preparation of poly(ethylene naphthalate), usually called PEN, is analogous to the preparation of $\mathrm{PET}^{51}$. The polymer in this case is obtained by reaction of 2,6-dimethylnaphthalene carboxilate with ethylene glycol (Scheme 9a). The process involves esterification reactions with the formation of 2-hydroxyethyl terminated oligomers, while the by-product methanol is removed to 
facilitate the progression of the reaction. The oligomers are then pre-polymerized at temperatures of $250-280{ }^{\circ} \mathrm{C}$ and any excess of ethylene glycol is removed. The pre-polymer formed is finally treated until polymerization is completed. Catalysts, such as manganese, zinc, calcium, cobalt and titanium are used to enhance the effectiveness of the esterification. Antimony is also frequently employed to favour polycondensation reactions. Although the process of production of PEN is similar to that of PET, the production of PEN requires more complex and more costly processes than PET. On the other hand, PEN has improved thermal, mechanical and gas barrier properties, which are derived from its inherent crystal liquid characteristics and that make it a suitable material for the production of fibres, films, special containers, etc.

\subsection{Phenol-Based Polymers}

Phenol and phenol derivatives are an important source of chemicals as described in section 5.2. In addition, phenols are widely used in the production of polycarbonates and phenolic resins.

\subsubsection{Polycarbonates}

An important family of phenol-based polymers is that of the polycarbonates, of which bisphenol A polycarbonate has the greatest economic impact ${ }^{69}$. In the past, this polymer was obtained by means of transesterification. However, this process has been replaced by an interfacial polycondensation process, which involves the reaction of bisphenol A (dissolved in aqueous phase as sodium bisphenolate) with phosgene (dissolved in organic phase, e.g., dichloromethane). The reaction occurs at the interface, giving rise to carbonate oligomers that then enter the organic phase. The presence of catalysts (e.g., triethylamine, tripropylamine, etc.) facilitates the subsequent polycondensation of the oligomers to yield the final polycarbonate (Scheme 10). 


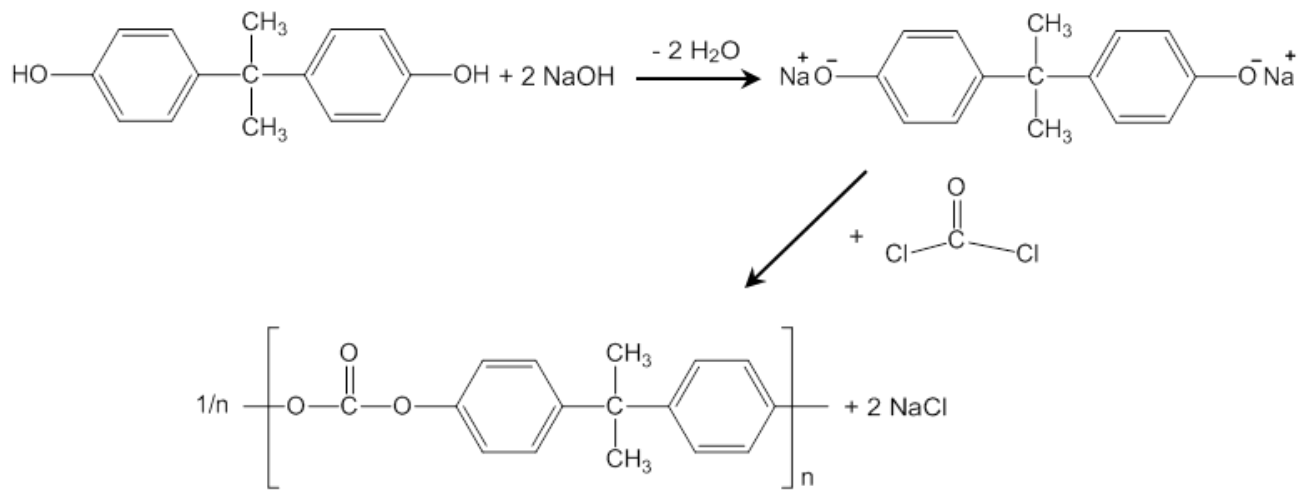

Scheme 10. Synthesis of bisphenol A polycarbonate by interfacial polycondensation.

Bisphenol A polycarbonate is a valuable polymer that combines extreme toughness with outstanding transparency and a strong resistance to distortion from heat. Therefore, it is commonly applied as a durable material in shatterproof windows, lightweight eyeglass lenses, data storage (e.g., CDs, DVDs, blue ray discs, etc.) and automobile applications, among others. It is usually commercialized under the trademark of $\operatorname{Lexan}^{70}$.

\subsubsection{Phenolic Resins}

Generally, phenolic resins are prepared from phenol (or a phenol derivative), an aldehyde and a catalyst $^{71,72}$ (Table 3). The most popular phenolic resins are those based on phenol and formaldehyde. In aqueous solutions, formaldehyde coexists in equilibrium with its methylene glycol isomer (Table 3). Depending on the catalyst used (acid or basic catalyst) the monomers react to form one of two main types of phenolic resins: Novolac resins (acid media) and Resol resins (basic media). 
Table 3. Representative phenols and aldehydes used for the production of phenolic resins.

Other Phenols Other aldehydes

In the case of the Novolac resins the reaction is carried out in the presence of an acid catalyst (e.g., sulphuric acid, sulfonic acid, oxalic acid and, occasionally, phosphoric acid) at a formaldehyde to phenol molar ratio of $<1^{71,72}$. In a first step, methylene glycol is converted into the corresponding hydrated carbonium, which reacts with phenol in ortho and para positions to give rise to o- and p-benzylic carbonium ions (Scheme 11a). In a second step, the benzylic carbonium ions react with a second molecule of phenol to produce o-o-, o-p- and p-p-diphenol (dimer) with a methylene bridge (Scheme 11b). The polymerization proceeds via any of the three 
a)

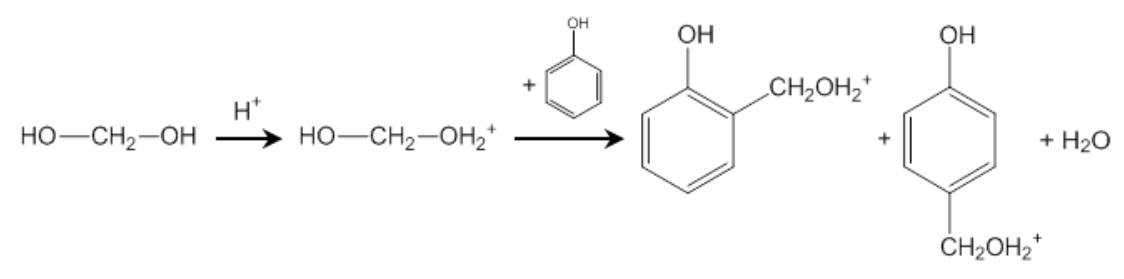

b)

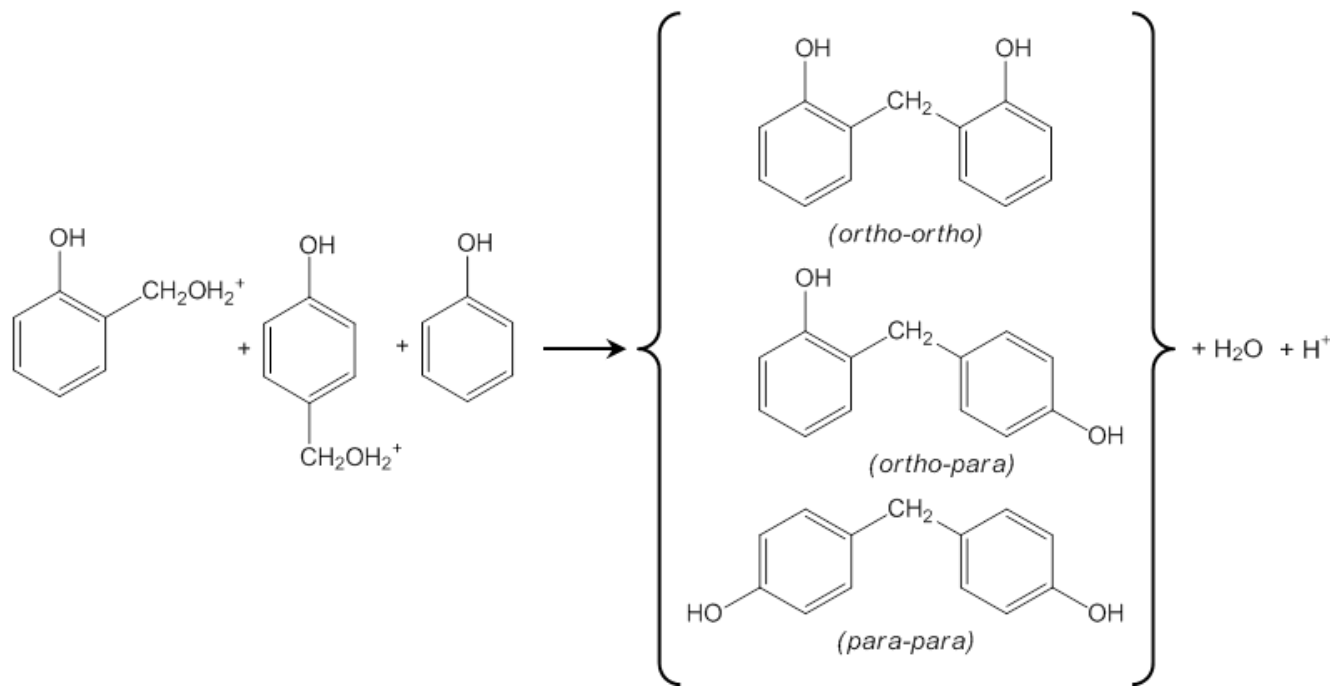

Scheme 11. Synthesis of monomers from phenol and formaldehyde for the manufacture of Novolac resin.

ortho and para positions of the aromatic rings, which makes it necessary to control the reactions in order to tailor the polymerization. A typical Novolac resin consists of several phenol units with a molecular weight of a few thousand of amu. It is interesting to note that one of the dimers resulting from the reaction of phenol with formaldehyde (bis(4-hydroxydiphenyl)methane, usually called bisphenol F) is also used as a monomer in the production of epoxy resins.

Resol resins are prepared in a basic medium (e.g., sodium hydroxide, calcium hydroxide and barium hydroxide) with formaldehyde to phenol molar ratios of $>1^{71,72,73}$. The reaction between formaldehyde and phenol is from an ortho position and this produces methylol phenol (Scheme 12a). This alcohol then reacts with itself to form a methylol phenolic or dibenzyl ether (Scheme 12b). Methylol phenol may also react with a second molecule of phenol to generate a dimer, in 
a)

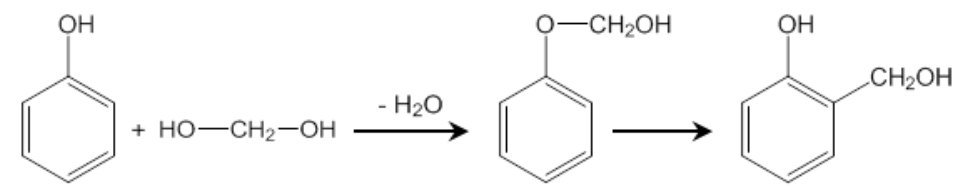

b)

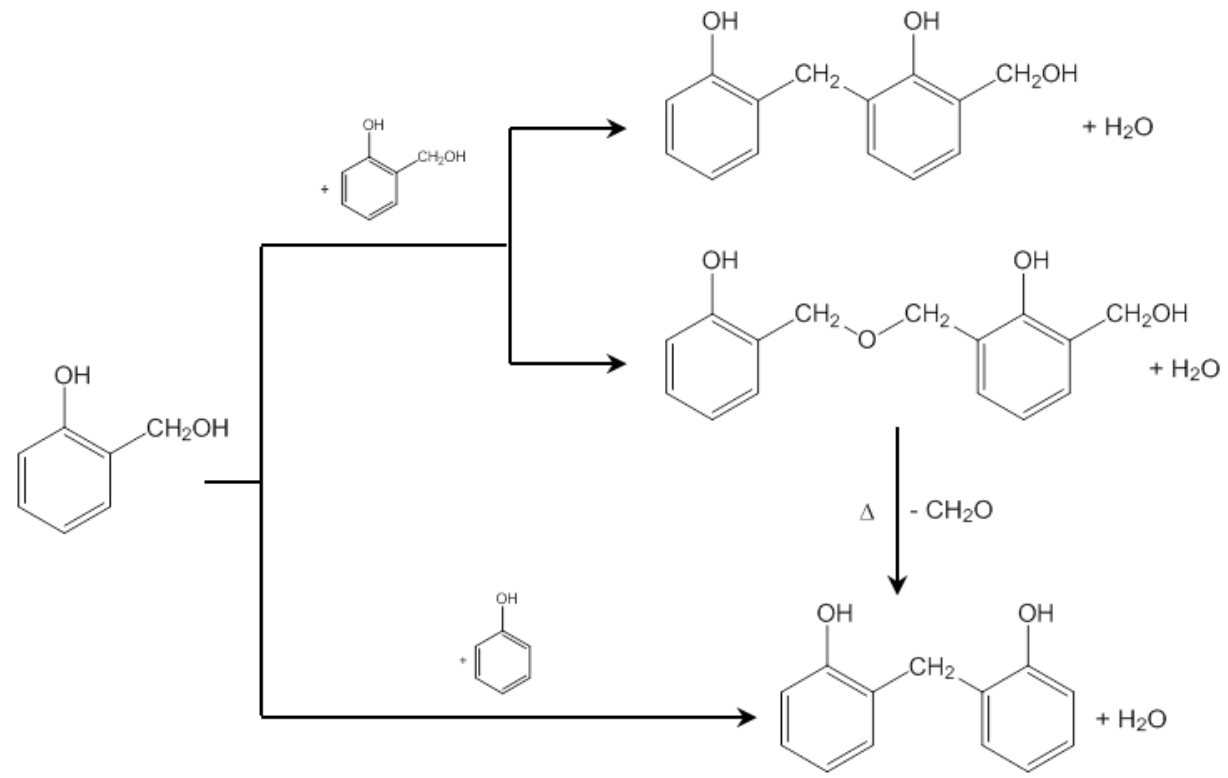

Scheme 12. Synthesis of monomers from phenol and formaldehyde for the manufacture of Resol resin.

which the phenols are joined by a methylene bridge (Scheme 12b). The polymerization proceeds to give rise to a highly cross-linked polymer. An interesting feature in the production of Resol resins is that small variations in parameters such as the formaldehyde:phenol molar ratio, $\mathrm{pH}$, catalyst type, reaction temperature, reaction time, etc., allow resins with different structures and characteristics to be obtained.

In general terms, phenolic resins show good adhesion properties, which make them suitable for use as a matrix of a large variety of organic and inorganic fillers and reinforcements. Moreover, they tolerate high temperatures and they are chemically resistant. For these reasons, phenolic resins are used in many technological fields such as the manufacture of composites, wood 
adhesive composites, foam, mineral insulation binders, laminates, friction and photoresistat

components, etc.

Table 4 summarizes the main properties and applications of the polymers described in this section.

Table 4. Polymers obtained from coal coking chemicals.

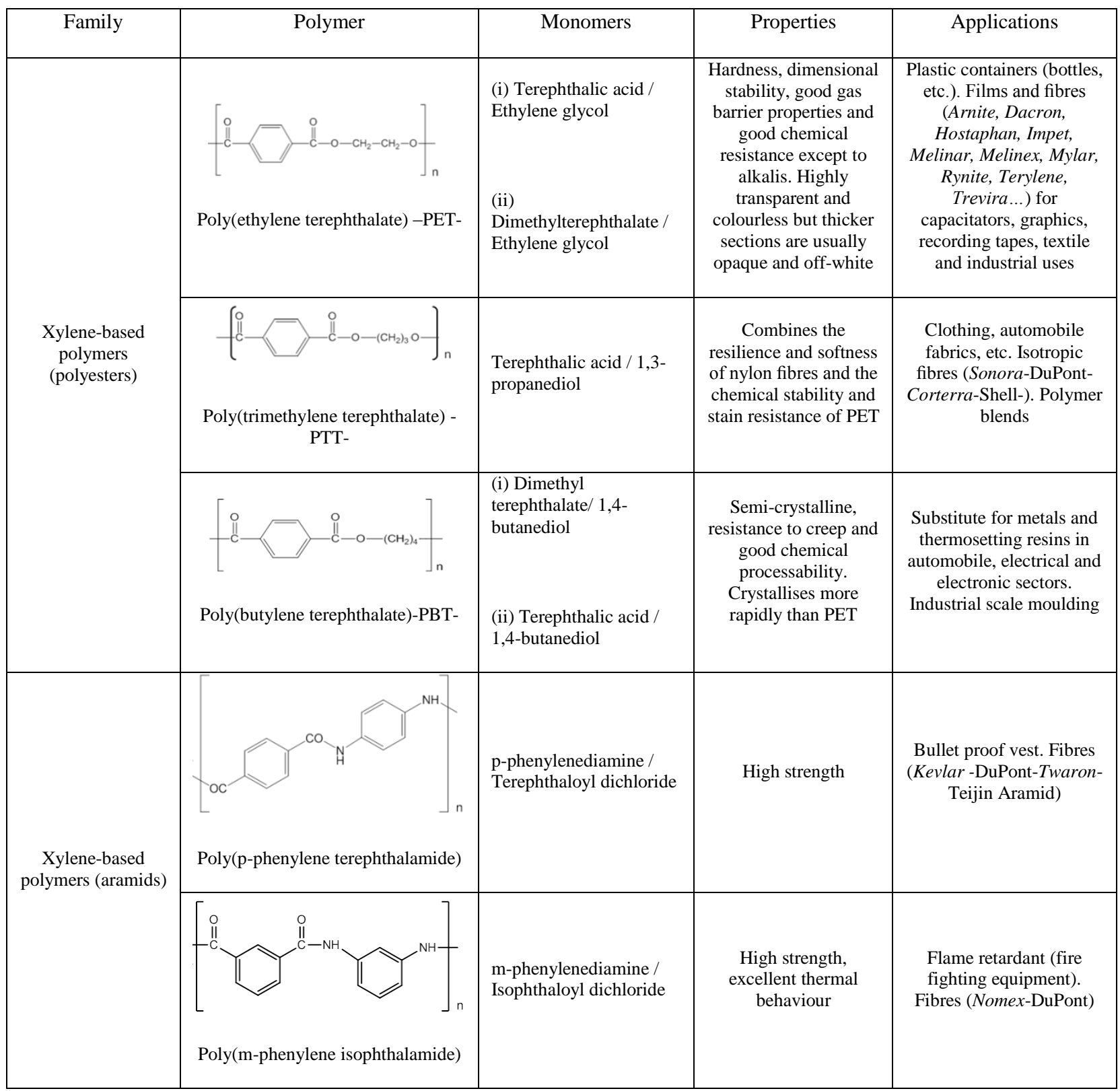




\begin{tabular}{|c|c|c|c|c|}
\hline $\begin{array}{c}\text { Xylene-based } \\
\text { polymers } \\
\text { (benzobisoxazoles) }\end{array}$ & $\begin{array}{c}\text { Poly(p-phenylene-2,6- } \\
\text { benzobisoxazole) -PBO- }\end{array}$ & $\begin{array}{l}\text { Bis(o-aminophenol) } \\
\text { derivatives / Aromatic } \\
\text { diacides or aromatic } \\
\text { dicarboxylic analogues }\end{array}$ & $\begin{array}{l}\text { Extremely high } \\
\text { mechanical properties } \\
\text { (higher than Kevlar). } \\
\text { Poor solubility }\end{array}$ & $\begin{array}{l}\text { High performance } \\
\text { applications (high cost). } \\
\text { Fibres (Zylon, Toyobo } \\
\text { Co.) }\end{array}$ \\
\hline \multirow{3}{*}{$\begin{array}{c}\text { Naphthalene-based } \\
\text { polymers }\end{array}$} & $\begin{array}{l}\text { Poly(trimethylene naphthalate) - } \\
\text { PTN- }\end{array}$ & \multirow{3}{*}{$\begin{array}{l}\text { 2,6-dimethylnaphthalene } \\
\text { carboxylate / Glycol } \\
\text { (propylene glycol, } \\
\text { butylene glycol or } \\
\text { ethylene glycol) }\end{array}$} & Gas barrier & $\begin{array}{l}\text { Fibres and resins. } \\
\text { Polymer blends }\end{array}$ \\
\hline & Poly(butylene naphthalate) $-\mathrm{PBN}-$ & & Gas barrier & $\begin{array}{l}\text { Fibres and resins. } \\
\text { Polymer blends }\end{array}$ \\
\hline & Poly(ethylene naphthalate)-PEN- & & $\begin{array}{c}\text { Crystal liquid, } \\
\text { improved thermal, } \\
\text { mechanical and gas } \\
\text { barrier properties than } \\
\text { PET }\end{array}$ & $\begin{array}{l}\text { Similar to PET. Fibres, } \\
\text { films, containers, etc. } \\
\text { Polymer blends }\end{array}$ \\
\hline $\begin{array}{l}\text { Phenol-based } \\
\text { polymers } \\
\text { (polycarbonates) }\end{array}$ & Bisphenol A polycarbonate & Bisphenol A / Phosgene & $\begin{array}{l}\text { Extreme toughness, } \\
\text { outstanding } \\
\text { transparency, strong } \\
\text { resistance to distortion } \\
\text { by heat }\end{array}$ & $\begin{array}{l}\text { Shatterproof windows, } \\
\text { lightweight eyeglass } \\
\text { lenses, data storage (e.g. } \\
\text { CDs, DVDs, Blue ray } \\
\text { discs, etc.), automobile } \\
\text { applications, etc. } \\
\text { (LEXAN- SABIC } \\
\text { Innovative Plastics) }\end{array}$ \\
\hline \multirow[t]{2}{*}{$\begin{array}{c}\text { Phenol-based } \\
\text { polymers (phenolic } \\
\text { resins) }\end{array}$} & Novolac resins (acid media) & $\begin{array}{l}\text { Formaldehyde / Phenol } \\
(\text { ratio }<1 \text { ) and acid } \\
\text { catalyst }\end{array}$ & \multirow{2}{*}{$\begin{array}{l}\text { Good adhesion } \\
\text { properties. Hardness } \\
\text { and heat and chemical } \\
\text { resistance properties. } \\
\text { Ability to withstand } \\
\text { high temperature } \\
\text { under mechanical load } \\
\text { with minimal } \\
\text { deformation or creep }\end{array}$} & \multirow{2}{*}{$\begin{array}{l}\text { Organic and inorganic } \\
\text { fillers and reinforcements } \\
\text { Manufacture of } \\
\text { composites, wood } \\
\text { adhesive, foam, mineral } \\
\text { insulation binders, } \\
\text { laminates, friction and } \\
\text { photoresistant } \\
\text { components }\end{array}$} \\
\hline & Resol resins (basic media) & $\begin{array}{l}\text { Formaldehyde / } \\
\text { Phenol (ratio >1) and } \\
\text { basic catalyst }\end{array}$ & & \\
\hline
\end{tabular}

\section{SYNTHETIC PITCHES FROM COAL COKING CHEMICALS}

Coal-tar pitch is traditionally used for the production of carbon materials mainly related to aluminium and steel industries. Moreover, the properties of commercial coal-tar pitches can be optimized for the preparation of carbon materials for high-technology applications (e.g., carbon 
fibres, carbon-carbon composites, etc.). Synthetic pitches with different specifications can also be obtained from other tar distillation fractions, resulting in single aromatics or mixtures of aromatics, to generate carbon materials with different microstructures, morphologies and/or $\operatorname{architectures}^{46}$. The synthesis of these carbon precursors involves the polymerizationcondensation of aromatics through controlled thermal treatments depending on the final material required. Developments in these areas, however, are mostly at laboratory level.

\subsection{The carbonization Process. Mechanisms}

Polycyclic Aromatic Hydrocarbons (PAH) when heated in an inert atmosphere, pass through a liquid crystal phase stage (or more specifically, a pseudo-liquid crystal phase) to yield a pregraphitic crystalline structure through a process called "carbonization". As it is an intermediate phase, this liquid crystal phase is known as carbonaceous mesophase ${ }^{74}$. From the chemical standpoint, the transformation of aromatics into mesophase and, subsequently, into graphitizable materials occurs by means of a dehydrogenative polymerization process (the polymerization in this case does not occur via the periodic repetition of single monomer units).

In the initial stages of the carbonization process, a series of reactions that lead to large molecules with a higher degree of aromatization take place ${ }^{21}$. These reactions mainly involve C-H and C-C bond cleavage to produce free radicals, molecular re-arrangements, thermal polymerization, aromatic condensation, the elimination of side chains and small molecules (e.g., $\mathrm{H}_{2}$ ), etc. Although from a mechanistic point of view these processes can be considered separately, they may occur simultaneously during carbonization. As a result of all these reactions, planar aromatic macromolecules with a lamellar and ordered structure (mesogens) are formed (Figure 6). Mesogens group together in parallel stacks by means of Van der Waals forces. These stacks 


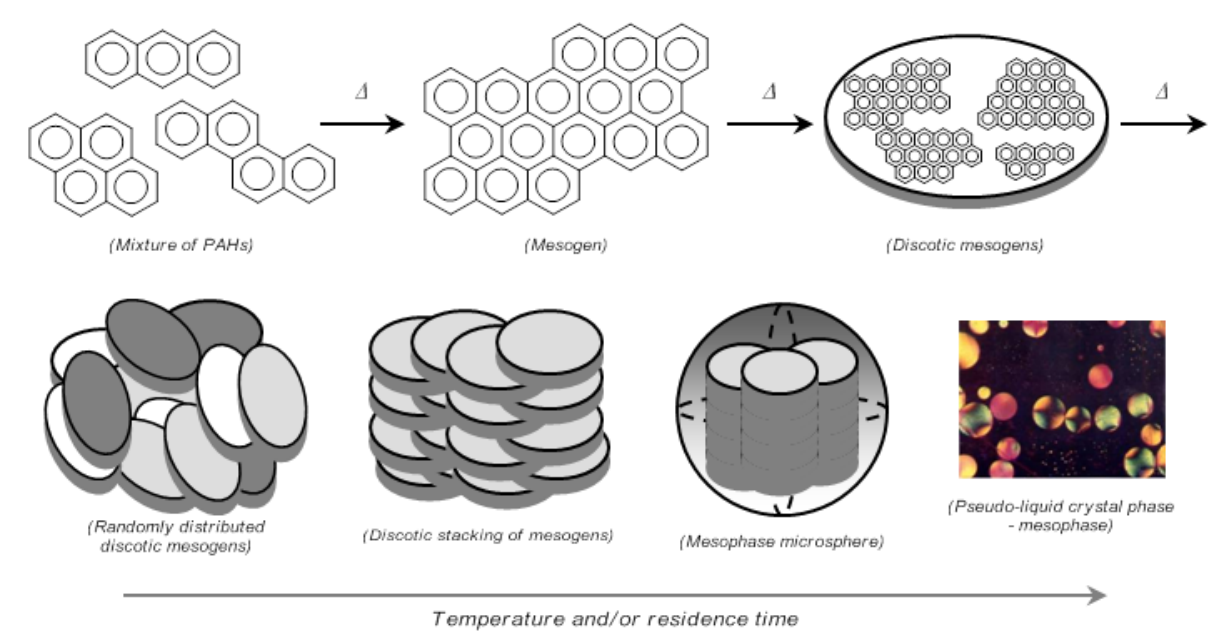

Figure 6. Schematic illustration of mesophase development from mixtures of PAHs.

segregate from the liquid isotropic phase to form small microspheres, which are optically anisotropic and constitute the carbonaceous mesophase. These microspheres are a discotic, nematic (thread-like) pseudo-liquid crystal phase. The formation of mesophase occurs as a spontaneous and homogeneous process within the isotropic phase when the attractive Van der Waals forces between the planar aromatic macromolecules overcome the dispersive forces originated by thermal agitation. In the initial stages of mesophase formation, these microspheres exhibit a thermotropic behaviour. However, as the temperature and/or residence time increase, the microspheres grow either via the incorporation of mesogens from the isotropic phase and/or via the coalescence of already formed microspheres ${ }^{75}$. The growth and coalescence of mesophase continues until the viscosity of the system is too high for it to be a liquid plastic phase and the mesophase solidifies, giving rise to a carbon material (semi-coke). The development of mesophase is a crucial step in the preparation of graphitizable carbon materials because it establishes the pre-graphitic order. This structural order is further consolidated by heating to temperatures above $2,500{ }^{\circ} \mathrm{C}$, giving rise to a graphitic material. Thus control over the 
mesophase allows the synthesis of carbons with a predetermined structure and properties ${ }^{76}$. In this way, it is possible to prepare graphitizable carbons with a mosaic microstructure (e.g., regular coke with a high mechanical strength for use as the carbon anode matrix in the electrolytic production of aluminium) or graphitizable carbons with a highly oriented microstructure (e.g., needle coke with a high electrical conductivity and a low coefficient of thermal expansion). Moreover, the carbonization process can be interrupted at the stage of mesophase formation in order to obtain an anisotropic pitch ${ }^{77}$. In this case, the mesophase still retains its plastic properties, and therefore, it can be processed into graphitizable carbon materials with different morphologies and/or architectures (e.g., mesophase can be spun into fibres $^{78}$ or mesophase can be moulded into preforms to produce self-sintering polygranular graphites $\left.^{79}\right)$.

\subsection{Pitches from Single Polycyclic Aromatic Hydrocarbons}

In addition to tar distillation, there are other routes for obtaining pitches through the polymerization of single PAHs and mixtures of PAHs. The transformation of PAHs into a pitchlike material involves reactions that lead to more condensed aromatic structures. The smaller amount of heteroatom-containing functional groups causes the transformation of these types of compounds to proceed in a different way to that of polymers, such as polyesters. The molecular growth in the polymerization of PAHs usually occurs via thermal condensation reactions which lead to planar macromolecules, as shown in section 7.1. Methylene or direct bridges between monomers are not common in these cases.

Several research studies have been carried out in order to determine the feasibility of transforming single PAHs (e.g., naphthalene, methylnapthalenes, anthracene, quinoline, etc.) into 

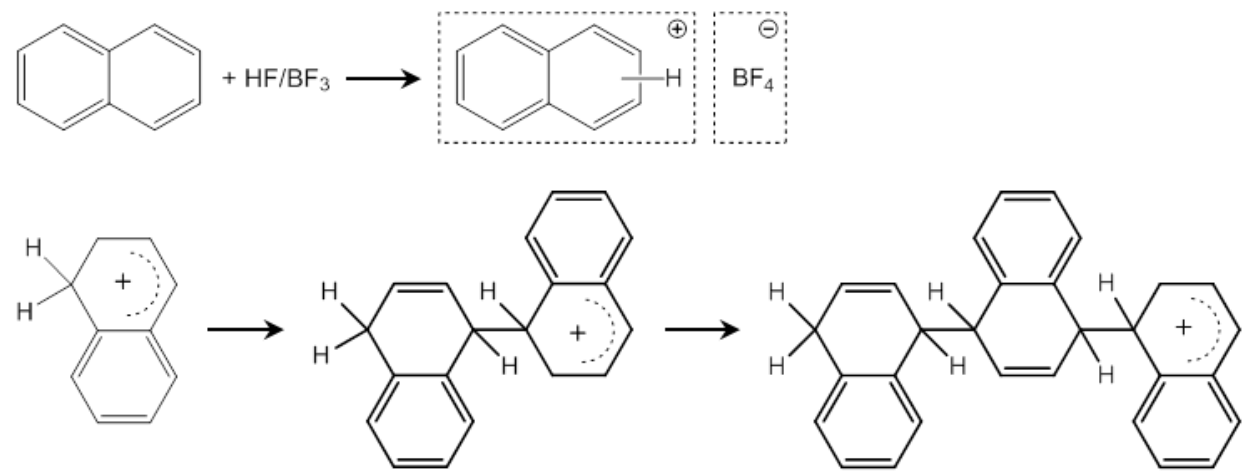

Scheme 13. Catalytic condensation of naphthalene with $\mathrm{HF} / \mathrm{BF}_{3}$.
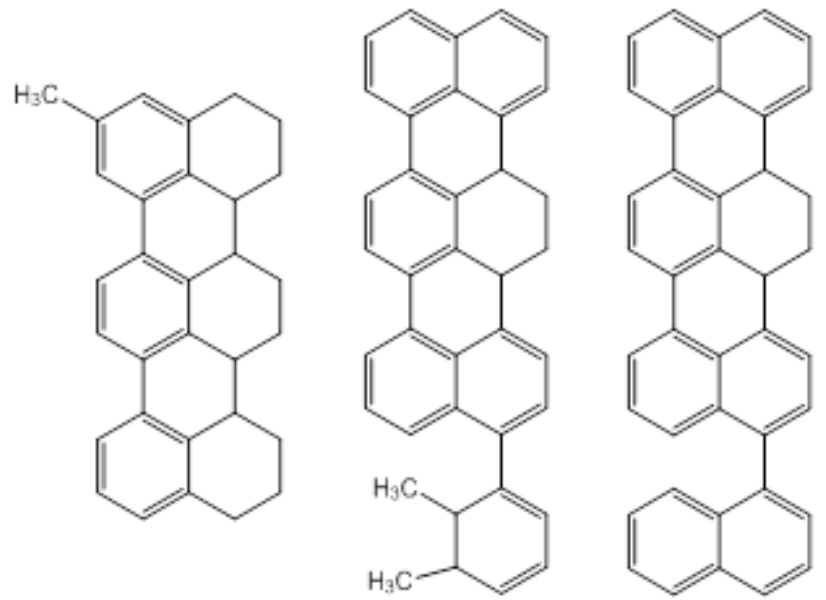

Scheme 14. Typical mesogen units in naphthalene-based pitch obtained by catalytic condensation with $H F / B F_{3}$.

pitch-like materials ${ }^{80,81,82}$ with specific properties for use in different applications in the field of carbon materials (e.g., carbon fibres, graphites, cokes with highly oriented microstructure, etc.). Among the single PAHs investigated, naphthalene has attracted most attention ${ }^{83}$. The polymerization of naphthalene requires the use of catalysts. Initial studies with aluminium trichloride have demonstrated that it is an effective Friedel-Crafts catalyst for the nondehydrogenative polymerization of single $\mathrm{PAHs}^{84}$. However, this catalyst has the drawback that it cannot be recycled, because when it is removed from the polymerized PAH (pitch-like 
material), usually by acid washing, it forms aluminium hydroxide. Moreover, aluminium trichloride is never totally removed from the pitch-like material and, although it is present only in small traces $(<10 \mathrm{ppm})$, it restricts the use of the pitch-like material as a precursor of carbons (e.g., the melt-spinning to produce carbon fibres) because some aluminium hydroxide particles remain in the pitch even after acid washing ${ }^{82}$. As an alternative solution, $\mathrm{HF} / \mathrm{BF}_{3}$ has been successfully applied as a Friedel-Crafts catalyst for the polymerization of naphthalene because $\mathrm{HF}$ and $\mathrm{BF}_{3}$ have low boiling points $\left(20\right.$ and $-101{ }^{\circ} \mathrm{C}$, respectively) and, consequently, both can be easily recovered from the pitch by atmospheric distillation and recycled for further use. $\mathrm{HF} / \mathrm{BF}_{3}$ reacts with naphthalene generating protonated complexes, which in turn react with more naphthalene to produce a dimer with two naphthenic hydrogens ${ }^{82}$ (Scheme 13). The polymerization progresses giving rise to oligomers (mesogens) made up of several naphthenic units either condensed or linked by single bridge bonds ${ }^{85}$ (Scheme 14). This preparation procedure is very versatile since it produces naphthalene-based pitches with different characteristics (e.g., isotropic or anisotropic pitches). Of the naphthalene-based pitches, ARA24 (mesophase pitch) has had the greatest impact on the market. This pitch has been industrially produced by Mitsubishi Gas Chemical and used as a precursor of high performance carbon fibres.

\subsection{Pitches from Mixtures of Polycyclic Aromatic Hydrocarbons}

The co-polymerization of synthetic mixtures of PAHs is of great importance for the preparation of pitch-like materials because of the benefits which can be obtained by combining the best characteristics of each PAH (lower environmental impact, improved fluidity, etc.). However, this practice has only been applied at laboratory scale and mainly for research purposes and/or the production of pitch-like materials on a small scale. On a large scale, industrial tar distilled 
fractions made of mixtures of PAHs are already in use. Among them, anthracene oil is perhaps the one that has attracted most interest ${ }^{42,86,87,88}$. This is because anthracene oil is a highly aromatic fraction that offers the possibility of co-polymerizing compounds of a large molecular size (3-5 aromatic rings) into a graphitizable material. The polymerization of anthracene oil is not possible under normal conditions (i.e., by heating at atmospheric pressure). This is because the components of anthracene oil are thermally stable at temperatures below their boiling point. Therefore, it is necessary to apply strategies that allow the polymerization of the components of anthracene oil before they are distilled. Some studies have demonstrated that aluminium trichloride and sulphur are effective catalysts for polymerizing anthracene oil, although aluminium trichloride has the limitation mentioned above (section 7.2), while sulphur has the drawback that it is incorporated in the molecular structure of polymerized compounds, which means that there is an excess of sulphur in the final products ${ }^{86,87}$. Similar effects to those caused by the presence of sulphur can be achieved by means of oxygen, but with the added benefit that oxygen only acts as a promoter of polymerization, after which it is eliminated from the molecular structure of the resultant moieties. Studies carried out in the presence of air at moderate temperatures $\left(<350^{\circ} \mathrm{C}\right)$ under pressure, have led to the polymerization of anthracene oil to yield a pitch-like material ${ }^{42}$. It is not easy to establish a mechanism for the oxidative thermal condensation of anthracene oil. However, gas chromatography studies have evidenced that PAHs containing aliphatic hydrogen are the most reactive (e.g. dihydroanthracene, acenapththene, fluorene, etc. $)^{89,90}$. Various mechanisms seem to contribute to the polymerization of anthracene oil: on the one hand, compounds containing $-\mathrm{CH}_{2}$ - that may give rise to dimers and molecules bonded by methylene, ether, carbonyl, ester bridges and, on the other hand, compounds with no aliphatic hydrogen (e.g., phenanthrene, anthracene, pyrene, etc.), which react more slowly to 


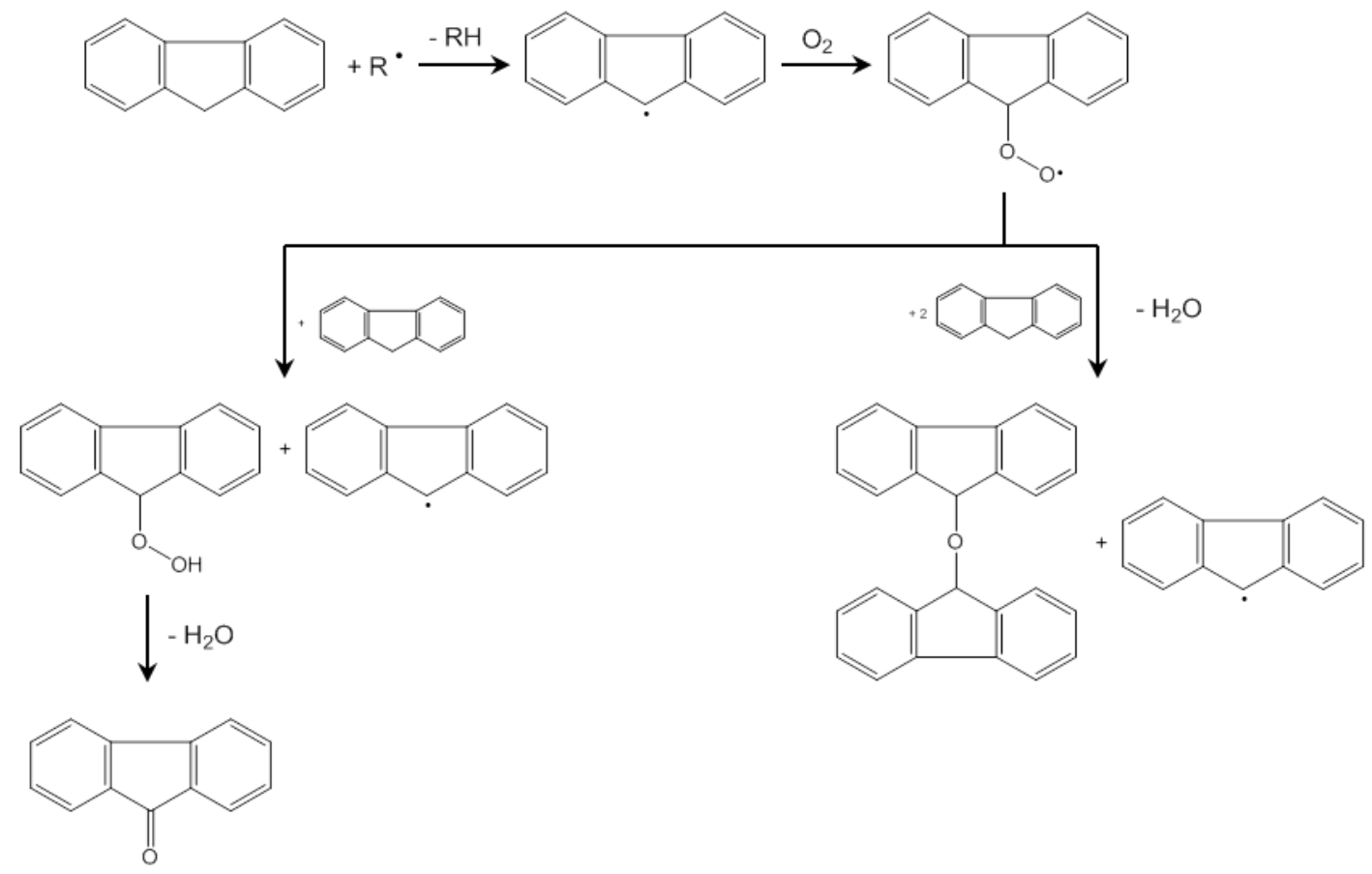

Scheme 15. Plausible mechanisms for the oxidation and condensation of fluorene.

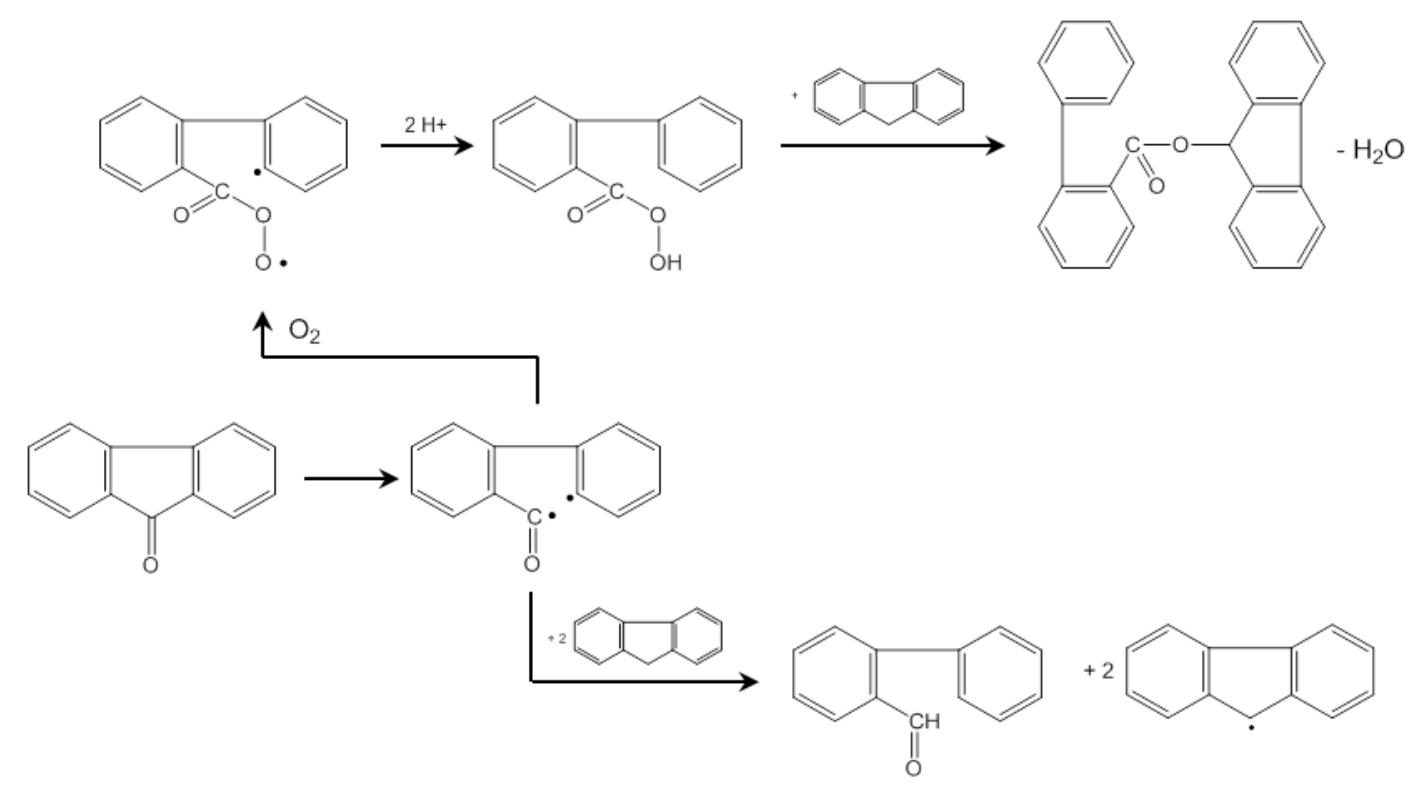

Scheme 16. Plausible mechanisms for the formation of fluorene-derived carbonyl functional groups.

give rise to dimers via the formation of oxiradicals. As an example, Scheme 15 shows plausible 
mechanisms of oxidation for the reaction of fluorene with oxygen. It is thought that oxidation occurs in steps ${ }^{91}$. Initially fluorene is oxidized to a fluorene-derived ketone or fluorene-derived ether. The ketone is then oxidized to an ester or aldehyde (Scheme 16) via the formation of a carbonyl radical acting as an intermediate ${ }^{89}$. On the other hand, oxidative thermal treatment may promote condensation reactions that lead to the formation of larger aromatic molecules ${ }^{80,92,93}$, as shown in Scheme 17.

If we consider the mechanisms proposed so far along with others that involve isomerization, molecular re-arrangement, etc., we can see that the operational conditions for the polymerization of anthracene oil not only affect the progression of the reactions but also the structure of the entities formed as intermediates, and, consequently, their thermal stability. The prevalence of one mechanism over another causes the reaction to lead to condensed planar macromolecules and/or to cross-linked oligomers. The latter structures are unable to generate graphitizable carbons on carbonization. However, cross-linked oligomers can be easily transformed into planar condensed

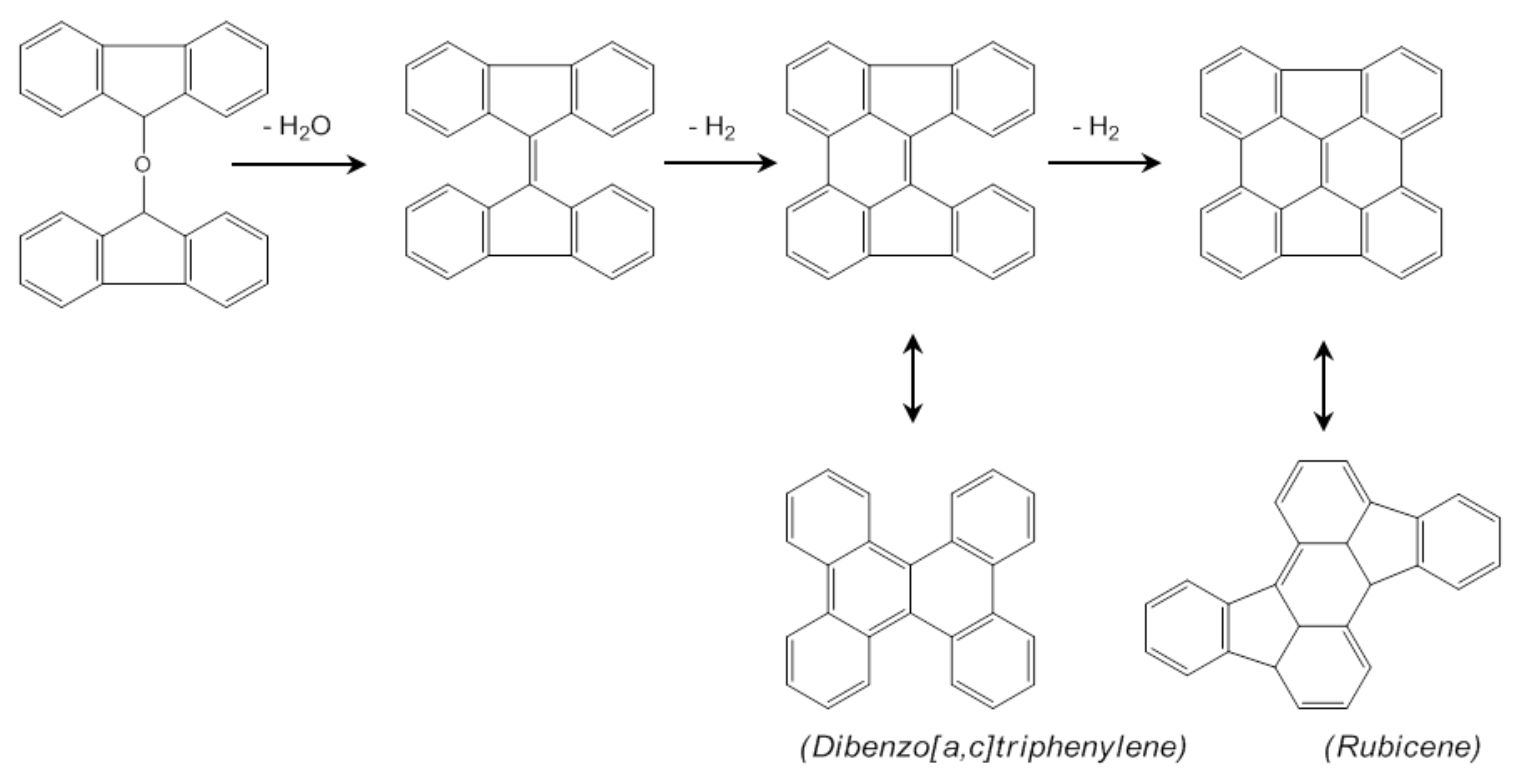

Scheme 17. Plausible mechanisms for the formation of aromatic macromolecules from fluorene-based aryl-ethers. 
a)

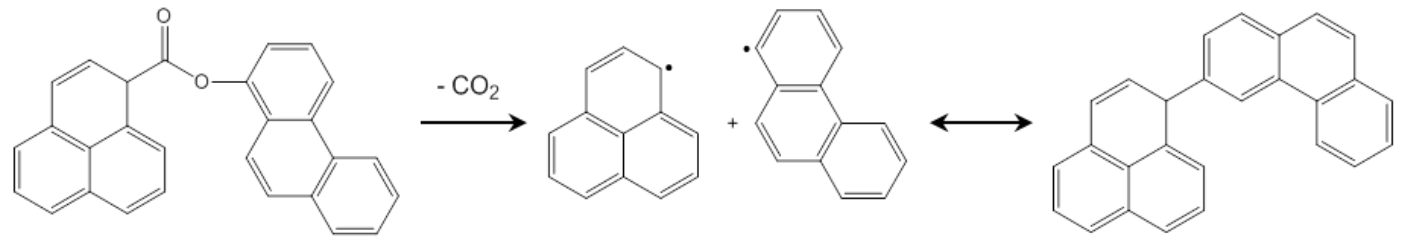

b)

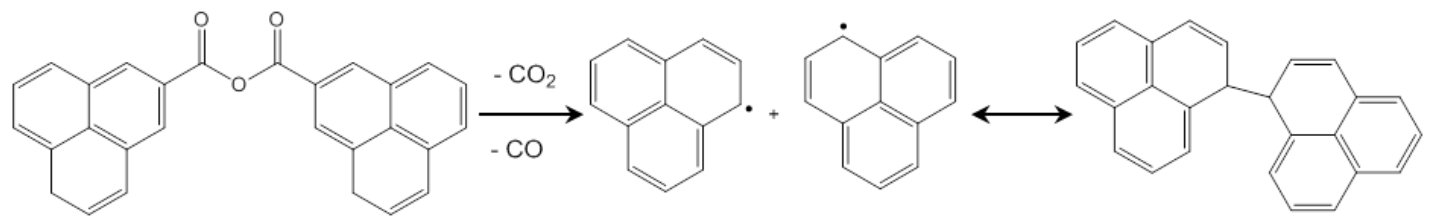

Scheme 18. Plausible mechanisms for the formation of aromatic molecules from (a) arylesters and (b) aryl-anhydrides.

macromolecules, when the oxygen content is at a low level, by thermal treatment in an inert atmosphere $^{88}$. As an example, Scheme 18 shows the progression of some oxygen functional groups towards the formation of bridged molecules, which with suitable thermal treatment will give rise to planar condensed macromolecules ${ }^{94}$.

This oxidative thermal process for polymerizing anthracene oil has already been successfully scaled up $^{95}$ and anthracene oil-based pitches have been produced at semi-industrial scale for different applications, both conventional (e.g., as binder and impregnating agents for the aluminium and steel-making industries) and advanced (e.g., as precursors of high-performance carbon fibres, polygranular graphites, graphene materials, etc.), Figure 7.

\section{FUTURE PROSPECTS}

Coal utilization is frequently portrayed as public enemy number one due to concerns over emissions of carbon dioxide and its contribution to global warming. Despite this poor image, 

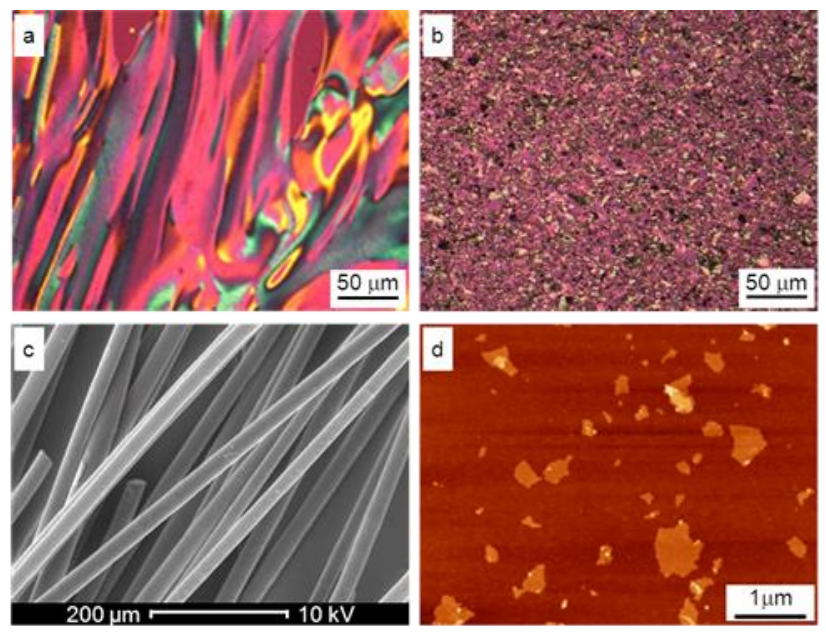

Figure 7. Optical microscopy images of anthracene oil-based (a) coke and (b) graphite; (c) SEM image of anthracene oil-based carbon fibres; (d) AFM image of anthracene oil-based graphene oxide.

world utilization of coal continues to rise, primarily because of the dominant role it plays in power generation.

Although new developments in the coking process and the use of coke in the blast furnace have improved the supply of coke, the demand for coking coal, and consequently coal tar, continues to increase. Today, China and the United States lead the world in the production of metallurgical coke (Figure 8$)^{96}$. Moreover, judging from the evolution of China in this field, it predominance is expected to increase. In fact, China has nearly tripled its production in the last decade, while in the United States it has remained almost constant. It is also worth noting that, whereas the production of metallurgical coke increased significantly in Australia and Asian countries (i.e., India and Indonesia) in the European countries, including Germany and Poland, it has declined. 


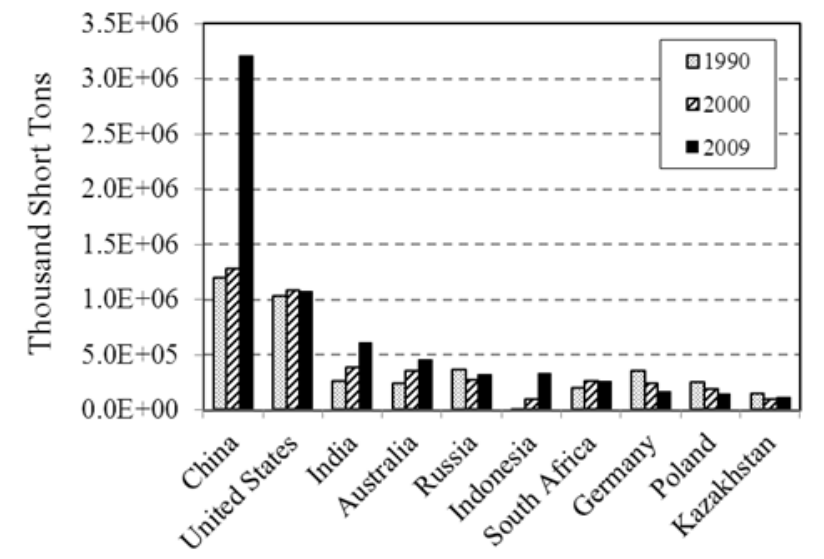

Figure 8. Evolution of metallurgical coke production in the ten major producer countries.

From the graphical data shown in Figure 8 and taking into account that the coking process yields $\sim 3-5$ wt. $\%$ of $\operatorname{tar}^{97}$, the supply of tar today and for the coming years seems to be guaranteed. This will favour the utilization of coal coking derivatives for the production of chemicals, if as predicted, crude oil becomes less available in the future.

Nevertheless, the importance of coal as a source of chemicals cannot be over emphasised. The organic chemical industry originally developed on the basis of the coal tar produced as a byproduct from the coking of coal to produce town gas, and later from the carbonization of coal for the production of metallurgical coke. At present time these by-products constitute only a small proportion of the feedstocks consumed by the chemical industry, but who is to say that in the not too distant future the wheel will not have turned full circle with coal carbonization again being the main source of the required chemicals.

Although these processes have largely been superseded by the oil-based petrochemical industry, the availability and security of supplies allied to the increasingly pressing economic 
considerations as oil supplies become less accessible suggest that, in the fullness of time the oftenvisaged "coalplex" could well become a reality. Moreover, some recent research has demonstrated the effectiveness of a novel catalyst, in the form of iron nanoparticles embedded in

carbon nanotubes, for converting carbon dioxide to a mixture of hydrocarbons ${ }^{98}$. Who is to say that such research will not lead to the chemical feedstocks of the future being produced from what are currently considered troublesome emissions of carbon dioxide from the various industrial applications of coal, including the coking industry and the utilisation of the coke so produced.

\section{AUTHORS' INFORMATION}

\section{Corresponding Author}

* Rosa Menendez. Instituto Nacional del Carbón, INCAR-CSIC. C/Francisco Pintado Fe 26. 33011-Oviedo, Spain. Tel. +34 985 119090. Fax +34 985 297662. E-mail: rosmenen@incar.csic.es

\section{Notes}

The authors declare no competing financial interest.

\section{Biographies}




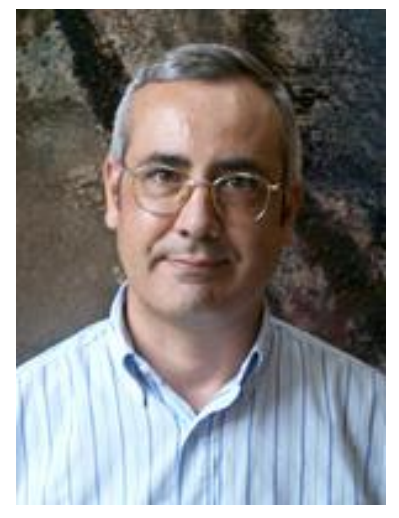

Marcos Granda was awarded his M.Sc. and Ph.D. in Chemistry from the University of Oviedo (Spain) in 1986 and 1992, respectively. After completing his Ph.D. degree, he spent two years in the University of Loughborough (Loughborough, UK). Currently, he is working in the Instituto Nacional del Carbón, INCAR-CSIC (Oviedo, Spain) as a research scientist. He has been Vicedirector of the INCAR-CSIC since 2013. His research is focused on the processing of coal and petroleum derivatives to produce carbon materials (fibres, graphites, graphenes, etc.).

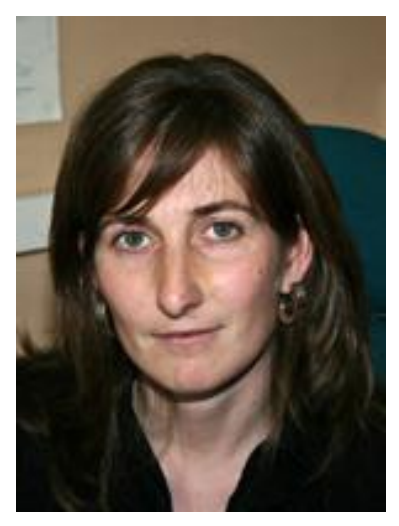

Clara Blanco was awarded her Ph.D. in Chemistry from the University of Oviedo (Spain) in 1998. Between 1999 and 2002 she worked for the University of Leeds (UK) as a postdoctoral fellow. In 2003 she obtained a permanent position in the Consejo Superior de Investigaciones Científicas at the Instituto Nacional del Carbon, INCAR-CSIC in Oviedo (Spain) where she is currently working. She has been Head of the Department of Chemistry of Materials since 2010. 
Her research is focused on the development of carbon precursors from coal and petroleum derivatives to produce different carbon materials (fibres, graphites, composites, graphenes, porous carbons for energy storage applications).

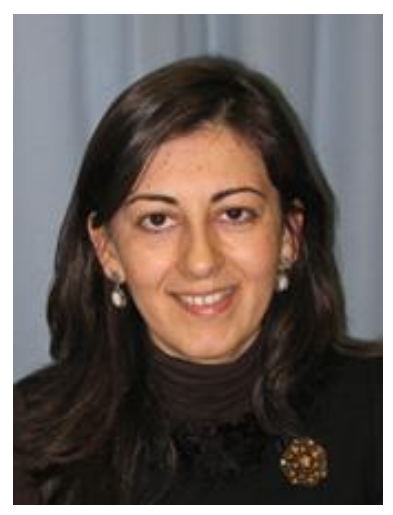

Patricia Alvarez is a Research Scientist of the Consejo Superior de Investigaciones Científicas (CSIC) at the Instituto Nacional del Carbon (INCAR) in Oviedo (Spain). She graduated in Chemistry from the University of Oviedo in 1997. In 2001 she obtained her Ph.D. degree from the same University. In 2002 she joined the Composites Group at INCAR focusing her activity on the preparation and characterization of carbon materials from coal and petroleum derivatives. After 2006 she joined the Department of Chemical Engineering at the Imperial College London, where she undertook extensive research into the characterization and transformation of coal and petroleum fractions. Since 2009 she has been at INCAR-CSIC, researching into the preparation and characterization of carbon materials (cokes and fibers) and nanomaterials (nanotubes and graphenes) and their catalytic, environmental and energy applications. 


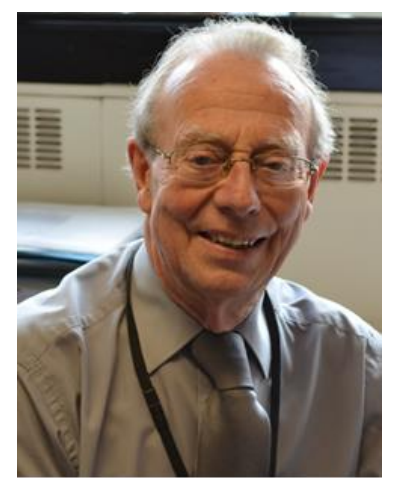

John W Patrick, B.Sc., Ph.D., C.Chem. FRIC. Graduated in Chemistry (London) and then obtained his Ph.D. (London) in Physics. Current interests include clean energy production and the reduction of carbon dioxide emissions. He worked initially for the National Coal Board, Scientific Services Department as Scientific Officer, then as Laboratory Manager of Nottingham Coal Survey Laboratory and later as Head of the Investigations Section in the Warwickshire Area Laboratory. He then joined the Fundamental Studies Section of the British Coke (later Carbonization) Research Association, eventually becoming Head of Fundamental Studies. He later moved to Loughborough University (Department of Chemical Engineering) as Director of the Carbon Research Group and Professor of Carbon Science, before moving in 2000 to the University of Nottingham (School of Chemical, Environmental and Mining Engineering) as Professor of Chemical Engineering and currently as Special Professor. A Member of the Editorial Board of Fuel between 1976 and 1980, he is now the Principal Editor of this journal. 


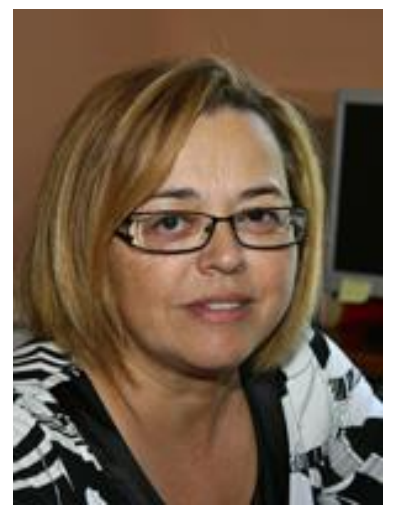

Rosa Menendez was awarded her Ph.D. in Chemistry from the University of Oviedo in 1986. She spent two years as postdoctoral fellow in the University of Newcastle upon Tyne (UK) and she has had stays at several Universities in USA (University of Southern Illinois at Carbondale, Clemson University) and Europe (Imperial College London, Nottingham University). In 2008 she gained a permanent position in the CSIC at the Instituto Nacional del Carbon (INCAR) in Oviedo (Spain) where she is now working as Research Professor. In the 90s she organized the Composites Group at INCAR, and she was director of the Institute for five years. She has been very active in R\&D management on a National and European level. Her main research activities are related with coal conversion processes, coal-based carbon precursors and the synthesis of carbon materials (fibres, composites, graphene, etc.) for different applications. She has been honoured with the 1996 Schunk Carbon Award and the XIX DuPont Award.

\section{ACKNOWLEDGMENTS}

The authors would like to express their gratitude to Dr. María A. Díez (Instituto Nacional del Carbón, CSIC), Dr. Juan J. Fernandez (Industrial Quimica del Nalon, S.A.) and Dr. Jose G. de la Campa (Instituto de Ciencia y Tecnologia de Polimeros, CSIC) for their valuable contribution to the discussion on the coal coking process, tar distillation process and the production of polymers from coal coking chemicals, respectively. 


\section{REFERENCES}

${ }^{1}$ Speight, J.G. The Chemistry and Technology of Coal; CRC Press: Boca Raton (USA), 2013; p. 607.

${ }^{2}$ Speight, J.G. The Chemistry and Technology of Coal; CRC Press: Boca Raton (USA), 2013; p. 579 .

${ }^{3}$ Speight, J.G. The Chemistry and Technology of Coal; CRC Press: Boca Raton (USA), 2013; p. 717.

${ }^{4}$ Alvarez, R.; Díez, M.A. Sciences of Carbon Materials; Publicaciones de la Universidad de Alicante: Alicante (Spain), Eds.: Marsh, H; Rodríguez-Reinoso, F., 2000; p. 595.

${ }^{5}$ Song, C., Schobert, H.H. Fuel Process. Technol., 1993, 34, 157.

${ }^{6}$ Schobert, H.H., Song, C. Fuel, 2002, 81, 15.

${ }^{7}$ Samuel, P., Maity, S., Khan, S., Roy, S.C. J. Sci. Ind. Res. India, 2008, 67, 1051.

${ }^{8}$ Roberts, J. Coal Carbonization; Pitman \& Sons: London, 1927; p. 62.

${ }^{9}$ Newman, J.W.; Newman, K.L. Introduction to Carbon Technologies; Publicaciones de la Universidad de Alicante: Alicante (Spain), Eds.: Marsh, H; Heintz, E.A.; Rodríguez-Reinoso, F., 1997; p. 269.

${ }^{10}$ Franck, H.G.; Stadelhofer, J.W. Industrial Aromatic Chemistry; Springer-Verlag: Berlin, 1988; p. 362.

${ }^{11}$ Berkovitch, I. Coal-Energy and Chemical Storehouse; Portcullis Press: Redhill (England), 1978; Ch. 2.

${ }^{12}$ Murder, R.E. Chemistry of Coal Utilization - Supplementary Volume, John Wiley \& Sons: New York, Ed. Lowry H.H., 1963; p. 629.

${ }^{13}$ McNeil, D. Chemistry of coal Utilization - Second Supplementary Volume, John Wiley \& Sons: New York, Ed. Elliot, M.A., 1981; p. 1003.

${ }^{14}$ Franck, H.G.; Stadelhofer, J.W. Industrial Aromatic Chemistry; Springer-Verlag: Berlin, 1988.

${ }^{15}$ Loison, R.; Foch, P.; Boyer, A. Coke Quality and Production, Butterworths: Cambridge, 1989.

${ }^{16}$ Díez, M.A.; Alvarez, R.; Barriocanal, C. Int. J. Coal Geol. 2002, 50, 389.

${ }^{17}$ Patrick, J.W. Sci. Prog. Oxf. 1974, 61, 375.

${ }^{18}$ Marsh, H. $51^{\text {st }}$ Ironmaking Conference Proceedings, Toronto (Canada), 1992, Vol. 51, 569. 
${ }^{19}$ Van Krevelen, D.W. Coal - Typology, Physics, Chemistry, Constitution, Elsevier: Amsterdam, 1993.

${ }^{20}$ Lewis, I.C. Fuel 1987, 66, 1527.

${ }^{21}$ Lewis, I.C. Carbon 1982, 20, 519.

${ }^{22}$ Alvarez, R.; Barriocanal, C.; Canga, C.S.; Canga, J.S.; Díez, M.A.; Gayol, O.M.; Miyar, E.A. Chromatographia, 1989, 27, 611.

${ }^{23}$ Franck, H.G.; Stadelhofer, J.W. Industrial Aromatic Chemistry; Springer-Verlag: Berlin, 1988; p. 27.

${ }^{24}$ Menéndez, R.; Bermejo, J.; Figueiras, A. Sciences of Carbon Materials, Publicaciones de la Universidad de Alicante: Alicante (Spain), Eds.: Marsh, H; Rodríguez-Reinoso, F., 2000; p. 173.

${ }^{25}$ Karrer, P. Organic Chemistry; Elsevier: Amsterdam, 1950, p.923.

${ }^{26}$ Casal M.D.; Diez, M.A.; Alvarez, R.; Barriocanal, C. International Journal of Coal Geology, 2008, 76, 237.

27 Dominguez, A.; Alvarez, R.; Blanco, C.; Diez, M.A. Journal of Chromatography A, 1996, 719, 181.

${ }^{28}$ McNeil, D. Coal Carbonization Products; The British Carbonization Research Association, Chesterfield (England); 1975, Ch. 2, p. 17.

${ }^{29}$ Speight, J.G. The Chemistry and Technology of Coal; CRC Press: Boca Raton (USA), 2013; p. 391.

30 Gray, R.J; Krupinski, K.C. Introduction to Carbon Technologies, Publicaciones de la Universidad de Alicante: Alicante (Spain), Eds.: Marsh, H; Heintz, E.A.; Rodríguez-Reinoso, F., 1997; p. 329.

31 Zander, M. Introduction to Carbon Technologies, Publicaciones de la Universidad de Alicante: Alicante (Spain), Eds.: Marsh, H; Heintz, E.A.; Rodríguez-Reinoso, F., 1997; p. 425.

${ }^{32}$ Speight, J.G. The Chemistry and Technology of Coal; CRC Press: Boca Raton (USA), 2013; p. 499.

${ }^{33}$ Speight, J.G. The Chemistry and Technology of Coal; CRC Press: Boca Raton (USA), 2013; p. 193.

${ }^{34}$ Franck, H.G.; Stadelhofer, J.W. Industrial Aromatic Chemistry; Springer-Verlag: Berlin, 1988; p. 265.

${ }^{35}$ Franck, H.G.; Stadelhofer, J.W. Industrial Aromatic Chemistry; Springer-Verlag: Berlin, 1988; p. 155. 
${ }^{36}$ Weber, M.; Weber, M. Phenolic Resins: A Century of Progress, Springer-Verlag: Berlin, 2010, $1^{\text {st }}$ Edition, p. 9.

37 Talukder, M. Kirk-Othmer Encyclopedia of Chemical Technologies; J. Wiley \& Sons, 2000; CD.ROM Edition, Vol. 16 (Naphthalene derivatives), p. 610.

${ }^{38}$ Franck, H.G.; Stadelhofer, J.W. Industrial Aromatic Chemistry; Springer-Verlag: Berlin, 1988; p. 298.

${ }^{39}$ Rokade, Y.B.; Sayyed, R.Z. Rasayan J. Chem. 2009, 2 (4), 972.

40 Booth, G. Ullmann's Encyclopedia of Industrial Chemistry; Wiley -VCH: Weinheim (Germany), 2005; 6th Edition, Vol. 23 (Naphthalene derivatives), p 671.

${ }^{41}$ Franck, H.G.; Stadelhofer, J.W. Industrial Aromatic Chemistry; Springer-Verlag: Berlin, 1988; p 309.

${ }^{42}$ Fernández, AL.; Granda, M.; Bermejo, J.; Menéndez, R. Carbon 2000, 38, 1315.

43 Taylor, R. Introduction to Carbon Technologies, Publicaciones de la Universidad de Alicante: Alicante (Spain), Eds.: Marsh, H; Heintz, E.A.; Rodríguez-Reinoso, F., 1997; p. 167.

${ }^{44}$ Franck, H.G.; Stadelhofer, J.W. Industrial Aromatic Chemistry; Springer-Verlag: Berlin, 1988; p 343.

${ }^{45}$ Franck, H.G.; Stadelhofer, J.W. Industrial Aromatic Chemistry; Springer-Verlag: Berlin, 1988; p 346.

${ }^{46}$ Granda, M.; Blanco, C.; Santamaria, R. Recent Research Developments in Materials Science, Research SignPost: Trivandrum (India), Ed.: Pandalai, S.G., 2004; p. 105.

${ }^{47}$ Rose, N.C. J. Chem. Educ. 1967, 44 (5), 283.

${ }^{48}$ Franck, H.G.; Stadelhofer, J.W. Industrial Aromatic Chemistry; Springer-Verlag: Berlin, 1988 p 280.

49 Sheehan, R.J. Ullmann's Encyclopedia of Industrial Chemistry; Wiley -VCH: Weinheim (Germany), 2005; 6th Edition, Vol. 36 (Terephthalic acid, dimethyl terephthalate and isophthalic acid), p. 17.

${ }^{50}$ Weissermel K.; Arpe, H.J. Industrial Organic Chemistry, Wiley - VHC $3^{\text {rd }}$ Edition, Weinheim (Germany), 1997; p. 393.

${ }^{51}$ Lillwitz, L.D. Appl. Catalysis A: General 2001, 221, 337.

52 Millini, R.; Frigerio, F.; Bellussi, G.; Pazzuconi, G.; Perego, C.; Pollesel, P.; Romano, U. Journal of Catalysis 2003, 217, 298. 
${ }^{53}$ Köpnick, H.; Schmidt, M.; Brügging, W.; Rüter J.; Kaminsky, W. Ullmann's Encyclopedia of Industrial Chemistry; Wiley -VCH: Weinheim (Germany), 2005; 6th Edition, Vol. 28 (Polyesters), p. 623.

${ }^{54}$ Fujimoto, K.; Azuma, Y.; Hisada, T. U.S. Patent 7198846, 2007.

55 Tsukamoto, R.; Hashimoto, N.; Hoshi, T. U.S. Patent Application 20060020103, 2006.

${ }^{56}$ Liu, J.; Bian S-G.; Xiao, M.; Wang, S-J.; Meng, Y-Z. Journal of Appl. Polym. Sci. 2010, 115, 3401 .

${ }^{57}$ Devroede, J.; Duchateau, R.; Koning, C.E.; Meuldijk, J. Journal of Appl. Polym. Sci. 2009, $114,2427$.

${ }^{58}$ Devroede, J.; Duchateau, R.; Koning, C.E.; Meuldijk, J. Journal of Appl. Polym. Sci. 2009, $114,2435$.

59 Mera, H.; Takata, T. Ullmann's Encyclopedia of Industrial Chemistry; Wiley -VCH: Weinheim (Germany), 2005; 6th Edition, Vol. 17 (High-performance fibers), p. 573.

${ }^{60}$ Knijnerberg, A.; Bos, J.; Dingemans, T. Polymer 2010, 51, 1887.

${ }^{61}$ Zhang, C.; Shoji, Y.; Higashira, T.; Tsukuda, A.; Ochi, T.; Ueda, M. Polymer Chemistry 2011, 49,4725 .

${ }^{62}$ Wang, H-H.; Su, C-C. J. App. Pol. Sci. 1996, 61, 1087.

${ }^{63}$ Rossbach, R.V.; Oberlein, G. Handbook of Polymer Synthesis - Part B; Marcel Dekker, Inc.: New York, 1992; Ed. Kricheldorf, H.R., Ch. 19 (Thermostable Polyheterocyclics), p. 1197

${ }^{64}$ Imai, Y.; Maeda, Y.; Takeuchi, H.; Park, K.-H.; Kakimoto, M. A.; Kurosaki, T. J. Polym. Sci. Part A, Polym. Chem. 2002, 40, 2656.

${ }^{65}$ Kim, J.-H.; Lee, J. K. Bull. Korean Chem. Soc. 2001, 22, 999.

${ }^{66}$ Sun, Y-M.; Hsu, K-R.; Wang, C-S. Journal of Appl. Polym. Sci. 1998, 67, 2245.

${ }^{67}$ Sun, Y-M.; Shieh, J-Y.; Wang, C-S. Eur. Poly. J. 1997, 33 (3), 317.

${ }^{68}$ Jeong, Y.G.; Jo, W.H.; Lee, S.C. Fibers and Polymers 2004, 5 (3), 245.

69 Serini, V. Ullmann's Encyclopedia of Industrial Chemistry; Wiley -VCH: Weinheim (Germany), 2005; 6th Edition, Vol. 18 (Polycarbonates), p. 603.

${ }^{70}$ Schmitz, P.; Janocha, S. Ullmann's Encyclopedia of Industrial Chemistry; Wiley -VCH: Weinheim (Germany), 2005; 6th Edition, Vol. 14 (Films), p. 649.

${ }^{71}$ Kopf, P.W. Encyclopedia of Polymer Science and Technology, J. Wiley \& Sons, Inc. 2002; $3^{\text {rd }}$ Edition; Vol. 7 (Polycarbonates), p. 649. 
72 Hesse, W. Ullmann's Encyclopedia of Industrial Chemistry; Wiley -VCH: Weinheim (Germany), 2005; 6th Edition, Vol. 26 (Phenolic resins), p. 583.

${ }^{73}$ Aiba, H. Phenolic Resins: A Century of Progress, Springer-Verlag: Berlin, 2010, $1^{\text {st }}$ Edition, p. 139.

74 Brooks, J.D.; Taylor, G.H. Chemistry and Physics of Carbon; Marcel Dekker: New York, 1968, Vol. 4, p. 243.

${ }^{75}$ Brooks, J.D.; Taylor, G.H. Carbon 1965, 3, 187.

${ }^{76}$ Granda, M.; Santamaria, R.; Menendez, R. Chemistry and Physics of Carbon; Marcel Dekker: New York, 2003; Vol. 28, p. 263.

${ }^{77}$ Blanco, C.; Santamaría, R.; Bermejo, J.; Menéndez, R. Carbon 2000, 38, 517.

${ }^{78}$ Yoon, S-H.; Korai, Mochida, I. Sciences of Carbon Materials, Publicaciones de la Universidad de Alicante: Alicante (Spain), Eds.: Marsh, H; Rodríguez-Reinoso, F., 2000; p. 287.

${ }^{79}$ Fanjul, F.; Granda, M.; Santamaria, R.; Menenedez, R. Journal of Materials Science 2004, 39 (4), 1213.

${ }^{80}$ Fitzer, E.; Mueller, K.; Schaeffer, W. Chemistry and Physics of Carbon; Marcel Dekker: New York, 1968, Vol. 7, p. 237.

${ }^{81}$ Lewis, I.C. Carbon 1980, 18, 191.

${ }^{82}$ Mochida, I.; Korai, Y.; Ku, C-H.; Watanage, F.; Sakai, Y. Carbon 2000, 38, 305.

${ }^{83}$ Mochida, I.; Shimizu, K.; Korai, Y.; Fujiyama, S.; Otsuka, H.; Sakai, Y. Carbon 1991, 28, 311.

${ }^{84}$ Mochida, I.; Sone, Y.; Korai, Y. Carbon 1985, 23, 175.

${ }^{85}$ Korai, Y.; Nakamura, M.; Mochida, I.; Sakai, Y.; Fujiyama, S. Carbon 1991, $29,561$.

${ }^{86}$ Fernandez, A.L.; Granda, M.; Bermejo, J.; Menendez, R.; Bernad, P. Energy \& Fuels 1998, $12,949$.

${ }^{87}$ Fernandez, A.L.; Granda, M.; Bermejo, J.; Menendez, R. Carbon 1999, 37, 1247.

${ }^{88}$ Bermejo, J.; Fernandez, A.L.; Granda, M.; Rubiera, F.; Suelves, I.; Menendez, R. Fuel 2001, $80,1229$.

${ }^{89}$ Drbohlav, J.; Stevenson, W.T.K. Carbon 1995, 33, 693.

${ }^{90}$ Metzinger, T.; Hüttinger, K.J. Carbon 1997, 35, 885.

${ }^{91}$ Fernandez, A.L. Ph.D. Thesis, University of Oviedo, Oviedo (Spain), 1998, Ch. 6. 
${ }^{92}$ Lewis, I.C.; Singer, L.S. Carbon 1967, 5, 373.

${ }^{93}$ Lewis, I.C.; Singer, L.S. J. Phys. Chem. 1981, 85, 354.

${ }^{94}$ Drbohlav, J.; Stevenson, W.T.K. Carbon 1995, 33, 713.

${ }^{95}$ Fernandez, J.J.; Alonso, F. Light Metals 2004, 499.

${ }^{96}$ Encyclopedia of the Nations. Metallurgical Coke - Production - Coal - Energy Information Administration - Country Comparison (http://www.nationsencyclopedia.com/WorldStats/EIAcoal-production-metallurgical-coke.html).

${ }^{97}$ Lankford, W.T.; Samways, N.L.; Craven, R.F.; McGannon, H.E. The Making, Shaping, and Treating of Steel. Association of Iron and Steel Engineers: Pittsburgh, 1985, p. 142.

${ }^{98}$ Chemistry World; 2013, Vol. 10, No. 4, p. 31. 